\title{
Quantum Low-Density Parity-Check Codes
}

\author{
Nikolas P. Breuckmann $\circledast^{1, *}$ and Jens Niklas Eberhardt $\odot^{2, \dagger}$ \\ ${ }^{1}$ Department of Computer Science, University College London, London, WC1E 6BT, United Kingdom \\ ${ }^{2}$ Mathematical Institute, University of Bonn, 53115 Bonn, Germany
}

(Received 7 March 2021; revised 27 May 2021; published 11 October 2021)

\begin{abstract}
Quantum error correction is an indispensable ingredient for scalable quantum computing. In this Perspective we discuss a particular class of quantum codes called "quantum low-density parity-check (LDPC) codes." The codes we discuss are alternatives to the surface code, which is currently the leading candidate to implement quantum fault tolerance. We introduce the zoo of quantum LDPC codes and discuss their potential for making quantum computers robust with regard to noise. In particular, we explain recent advances in the theory of quantum LDPC codes related to certain product constructions and discuss open problems in the field.
\end{abstract}

DOI: 10.1103/PRXQuantum.2.040101

\section{INTRODUCTION}

Whenever quantum information is stored or manipulated, errors are bound to occur. While there has been tremendous progress toward the realization of quantum processors [1-3], the implementation of error-corrected quantum memories and the demonstration of scalable fault-tolerant quantum computations remain formidable challenges.

One reason why error correction has a prominent role in the realization of quantum computation is that qubits are inherently more fragile than classical bits. Another, subtler reason is that quantum computers mix the ana$\log$ (amplitudes) with the discrete (measurements). Analog computation, which is based on the manipulation of continuous variables rather than bits, should serve as a cautionary tale: while it is well known that, in theory, certain analog computers are vastly more powerful than even quantum computers [4], they remain fictional devices since there are no known error correction schemes that apply to the relevant analog computing architectures. It is thus unknown how to scale them to a size relevant for solving practically relevant tasks. This was already pointed out by early critics of quantum computers, who considered them to be nothing but analog computers in disguise [5,6]. Shor [7] countered this criticism by introducing the first quantum

\footnotetext{
*n.breuckmann@ucl.ac.uk

†mail@jenseberhardt.com
}

Published by the American Physical Society under the terms of the Creative Commons Attribution 4.0 International license. Further distribution of this work must maintain attribution to the author(s) and the published article's title, journal citation, and DOI. error correcting code in his foundational paper, showing that it is possible to protect quantum information. This established quantum error correction as a field and started the pursuit to find optimal quantum error correcting codes.

Shortly after Shor's work, Kitaev's toric code [8,9] and the related planar surface code $[10,11]$ were proposed. Surface codes are currently the leading approach for fault-tolerant quantum computation due to their high error correction threshold and planar layout. They exist for a variable number of physical qubits $n$, and encode a single logical qubit $k=1$. Their distance $d$, a measure for the error-correcting capability, scales with $\sqrt{n}$. It is likely that we will soon see the first realization of small instances of surface codes (see Ref. [12] for recent results and a historical overview of experimental progress).

However, the surface code family does not compare favorably with the best known families of classical codes, where $k$ and $d$ scale linearly with $n$. While there exist quantum codes that match the properties of classical codes $[13,14]$, they have a significant technical drawback: the parity checks, which have to be measured to infer the error, involve a growing number of physical qubits per logical qubit. This is an issue as arbitrarily large checks cannot be reliably facilitated without additional fault tolerance constructions. Furthermore, the measurement of the checks cannot be parallelized, leading to a buildup of errors due to idling qubits. Finally, many decoding algorithms are based on the assumption that the parity checks are sparse.

Similar issues arose in classical coding theory and were solved by low-density parity-check (LDPC) codes, where the number of bits involved in each check and the number of checks acting on each bit are bound by a constant for all members of the code family. LDPC codes have been very 
successful in the classical setting as they approach upper bounds due to Shannon on the amount of information that can be reliably transferred through a noisy channel $[15,16]$. Many modern technologies, such as WiFi, DVB-T, and 5G, are error-corrected by LDPC codes [17].

It is therefore natural to consider quantum LDPC codes, which are defined in the same way. While LDPC codes have been the subject of intense study in classical coding theory in the last few decades, their quantum analogs have only recently become the focus of attention. Much of the interest in LPDC quantum codes was spurred by Gottesman's remarkable result in 2013 [18] showing that quantum LDPC codes with a constant encoding rate can reduce the overhead of fault-tolerant quantum computation to be constant. This is in contrast to other quantum fault tolerance schemes, where to perform a longer computation, it is necessary to suppress errors further, which requires larger codes and thus a growing number of physical qubits $[8,19,20]$. Moreover, one might hope that quantum LDPC codes can approach channel capacity similarly to (classical) LDPC codes. We do not touch upon the challenging field of quantum channel capacities here; see Refs. [21-23] for references.

Classical coding theory is spoiled with constructions of LDPC codes with good properties. Taking random codes gives LDPC codes with a constant encoding rate $k / n$ and linear distance $d \propto n$ with high probability $[15,24]$. In comparison, it is much harder to construct quantum LDPC codes, and it is still a major open problem whether quantum LDPC codes exist that rival the parameters of their classical counterparts [25]. For example, surface codes and color codes, which are LDPC codes and are among the most studied quantum codes, encode only a low constant number of qubits and have $d \propto \sqrt{n}$ at best. There has been a lot of recent progress toward better LDPC codes, and several families of quantum LDPC codes have been constructed that significantly outperform surface codes and color codes in terms of their asymptotic parameters. This is a strong indication for their potential, although they have not received the same amount of attention as surface codes as of now.

Driven by this recent success, this Perspective surveys the exciting and emerging field of quantum LDPC codes. We discuss the construction and analysis of these novel codes, which involve ideas and challenges that go far beyond the theory of surface and color codes. The field still has many open questions of central importance that we highlight throughout the text.

First, we introduce some background on stabilizer and Calderbank-Shor-Steane (CSS) codes as well as their relation to homological algebra and geometry (Sec. II) for the convenience of the reader.

Next, we discuss constructions of quantum LDPC codes using the geometry of manifolds (Sec. III). We discuss various product constructions of quantum LDPC codes inspired by the topological notions of Cartesian products and fiber bundles, which are at the heart of recent breakthrough results (Sec. IV). An overview of the parameters of these codes can be found in Table I.

Somewhat paradoxically, we also review quantum codes that are strictly speaking not LDPC codes but that also yield high encoding rates and require some sort of nonlocality (Sec. V). We do not discuss surface and color codes in much detail since they have already been covered extensively in the literature; see Refs. [26-28].

Besides introducing the zoo of quantum LDPC codes, we discuss challenges and opportunities regarding their use for quantum error correction (Sec. VI). For example, we address decoding algorithms and the challenges in hardware implementation. We refrain from directly comparing different codes in terms of their thresholds due to the wide variety of error models and assumptions going into numerical simulations, but we do refer the reader to the relevant literature. We also discuss applications of quantum LDPC codes beyond quantum error correction and quantum fault tolerance (Sec. VII).

TABLE I. The best proven parameters of quantum LDPC codes discussed in this paper. Some entries refer to a whole method of constructing codes. In these cases we cite the best known proven parameters of a family quantum LDPC codes constructed using this method.

\begin{tabular}{lccc}
\hline \hline Name & $k$ & $d$ & Section \\
\hline Two-dimensional hyperbolic codes & $\Theta(n)$ & $\Theta(\log n)$ & III A \\
Four-dimensional hyperbolic codes & $\Theta(n)$ & $\Omega(\sqrt[10]{n})$ & III A \\
Freedman-Meyer-Luo codes & 2 & $\Omega(\sqrt[4]{\log n} \sqrt{n})$ & III B \\
Tensor products (good classical codes) & $\Theta(n)$ & $\Theta(\sqrt{n})$ & IV A \\
Tensor products (Ramanujan complexes) & $\Theta(\sqrt{n})$ & $\Omega(\sqrt{n} \operatorname{polylog} n)$ & IV A \\
Fibre bundle codes & $\Theta\left(n^{3 / 5} / \operatorname{polylog} n\right)$ & $\Omega\left(n^{3 / 5} / \operatorname{polylog} n\right)$ & IV B \\
Lifted product codes & $\Theta\left(n^{\alpha} \log n\right)$ & $\Omega\left(n^{1-\alpha} / \log n\right)$ & IV C \\
Balanced product codes & $\Theta\left(n^{4 / 5}\right)$ & $\Omega\left(n^{3 / 5}\right)$ & IV D \\
\hline \hline
\end{tabular}




\section{A. Notation and conventions}

All vector spaces, unless otherwise mentioned, are over the field with two elements $\mathbb{F}_{2}$. The notation $[n, k, d]$ describes the parameters of a classical binary code: number of bits $n$, number of encoded bits $k$, and minimum distance $d$. Similarly, we use the notation $[[n, k, d]]$ for quantum codes.

Often, the exact relation between the code parameters is not known. However, it is sometimes possible to make asymptotic statements for which we need the following notation. For two positive functions $f$ and $g$, we write $f \in O(g)$ if $\limsup _{n \rightarrow \infty} f(n) / g(n)<$ $\infty, f \in o(g)$ if $\lim _{n \rightarrow \infty} f(n) / g(n)=0, f \in \Omega(g)$ if $\liminf _{n \rightarrow \infty} f(n) / g(n)>0$, and $f \in \Theta(g)$ if $f \in O(g)$ and $f \in \Omega(g)$. The above symbols can be interpreted as sets of functions. However, whenever convenient, we abuse the notation and write expressions such as $f \leq O(g)$, with the obvious interpretation.

\section{BACKGROUND}

One exciting aspect of the theory of quantum codes is the fact that it draws from a diverse mathematical and physical background. It combines research from classical coding theory, systolic geometry, homology, and combinatorics. This manifests itself in several different perspectives that people from different areas have on quantum LDPC codes. We briefly survey these perspectives here for the convenience of the reader.

\section{A. Quantum codes}

For background on general quantum codes, we refer to the review by Terhal [29] and Preskill's lecture notes [30]. In this text, we focus on stabilizer codes, which are the most studied class of quantum codes; see Ref. [31].

\section{Stabilizer and CSS codes}

An $[[n, k, d]]$ stabilizer quantum code is defined by a commutative group $S$ that is a subgroup of the Pauli group acting on the state space of $n$ physical qubits $\left(\mathbb{C}^{2}\right)^{\otimes n}$ not containing $-I$. The group $S$ has $n-k$ independent generators, called "stabilizer checks." The code subspace is defined as the +1 eigenspace of $S$ and can be interpreted as the state space of $k$ logical qubits. The nontrivial logical operators on the code space correspond to the elements of the Pauli group that commute with $S$ but are not in $S$ themselves. The distance $d$ is the smallest number of physical qubits in the support of a nontrivial logical operator. A slight generalization of stabilizer codes is subsystem codes, where only some logical degrees of freedom are used, while the others are downgraded to become gauge qubits and their corresponding logical operators are called "gauge operators."

A CSS code is defined by a pair of classical linear binary codes $C_{X}, C_{Z} \subset \mathbb{F}_{2}^{n}$ such that the orthogonality condition
$C_{X} \subset C_{Z}^{\perp}$ is satisfied; see Ref. [32]. We assume that the codes $C_{X}$ and $C_{Z}$ are given by their parity-check matrices $H_{X}$ and $H_{Z}$. A CSS code defines a stabilizer code, where the stabilizer group is generated by the stabilizer checks $X^{c}=\prod_{i=1}^{n} X_{i}^{c_{i}}$, where $c$ is a row of $H_{X}$, and $Z^{d}=\prod_{i=1}^{n} Z_{i}^{d_{i}}$ where $d$ is a row of $H_{Z}$. The commutativity of the stabilizer group is ensured by the orthogonality condition $C_{X} \subset C_{Z}^{\perp}$, which is equivalent to

$$
H_{Z} H_{X}^{\mathrm{tr}}=0 \quad \bmod 2 .
$$

It is straightforward to express properties of the stabilizer code in terms of the CSS code. We focus mostly on CSS codes and note that this is a only a minor restriction since any $[[n, k, d]]$ stabilizer code can be mapped onto a [[4n, 2k,2d]] CSS code; see Ref. [33].

\section{Quantum LDPC codes}

Generally, we are not interested in individual quantum codes but rather are interested in families of codes with a growing number of physical qubits. By abuse of language we will often simply speak of $a$ code when we actually mean a family.

A LDPC code is a family of stabilizer codes such that the number of qubits participating in each check operator and the number of stabilizer checks that each qubit participates in are both bounded by a constant. For CSS codes this means that the Hamming weight of each row and column of $H_{X}$ and $H_{Z}$ is bounded by a constant.

More generally, one can define quantum LDPC codes to include codes that are defined by a set of commutative projectors in the obvious way; see Sec. VD. We consider subsystem codes to be LDPC codes if their stabilizer checks fulfill the LDPC condition.

A major open problem in quantum error correction is whether good quantum LDPC codes exist. "Good" is terminology from classical coding theory referring to the property of a code as having $k \in \Theta(n)$ and $d \in \Theta(n)$. Taking a sparse parity-check matrix at random defines good classical codes [24], but taking two random parity-check matrices to define $H_{X}$ and $H_{Z}$ of a quantum code does not work, as Eq. (1) will not be satisfied. Good quantum codes that are not LDPC codes have been known since the early days of quantum error correction [13, 14]. However, it was only in 2020 that quantum LDPC codes were constructed that have distances scaling as $d \geq$ $\Omega(\sqrt{n}$ polylog $n)$. Recently, there has been rapid progress on increasing the distance of quantum LDPC codes (see Sec. IV).

\section{B. Perspectives on CSS codes}

Besides their description in terms of parity-check matrices, there are other useful representations of CSS codes, which we briefly present in the following sections. 


\section{Chain complexes}

Soon after quantum codes were introduced by Shor, it was discovered that they can be constructed using tools from homological algebra [9-11,34]. We briefly show how this homological description is related to our earlier definition of CSS codes: we consider chain complexes $C$ of length $n+1$ that are collections of linear maps $\partial_{i}$ called "boundary operators" and $\mathbb{F}_{2}$-vector spaces $C_{i}$

$$
C=\left(C_{n} \stackrel{\partial_{n}}{\longrightarrow} \cdots \stackrel{\partial_{2}}{\longrightarrow} C_{1} \stackrel{\partial_{1}}{\longrightarrow} C_{0}\right)
$$

fulfilling $\partial_{i} \partial_{i+1}=0$. Moreover, we assume that all vector spaces $C_{i}$ are equipped with a basis such that the boundary operators $\partial_{i}$ can be interpreted as matrices.

Chain complexes come from algebraic topology (see Sec. II B 3), and are hence described in topological terms. For example, the basis vectors of $C_{i}$ are called " $i$-cells" and elements are called " $i$-chains." An $i$-cycle is an $i$ chain with a trivial boundary and so an element in $\operatorname{ker}\left(\partial_{i}\right)$, whereas an $i$-boundary is an $i$-chain in the image of the boundary operator and so an element in $\operatorname{im}\left(\partial_{i+1}\right)$. The $i$ th homology $H_{i}(C)=\operatorname{ker} \partial_{i} /$ im $\partial_{i+1}$ of $C$ is the vector space of $i$-cycles modulo $i$-boundaries. Dually, one defines $i$-cocycles, $i$-coboundaries, and the $i$ th cohomology $H^{i}(X)=\operatorname{ker} \partial_{i+1}^{\mathrm{tr}} / \operatorname{im} \partial_{i}^{\mathrm{tr}}$.

In this homological language, a classical code corresponds to a chain complex of length 2 via its parity-check matrix, while a CSS code can be represented by a chain complex of length 3 :

$$
C=\left(C_{2} \stackrel{\partial_{2}=H_{Z}^{t r}}{\longrightarrow} C_{1} \stackrel{\partial_{1}=H_{X}}{\longrightarrow} C_{0}\right) .
$$

With this correspondence, the logical $Z$-operators correspond to the homology group $H_{1}(C)=\operatorname{ker} \partial_{1} / \mathrm{im} \partial_{2}$ and the logical $X$-operators correspond to the cohomology group $H^{1}(C)=\operatorname{ker} \partial_{2}^{\text {tr }} / \mathrm{im} \partial_{1}^{\text {tr }}$. The number of logical qubits $k=\operatorname{dim} H_{1}(C)=\operatorname{dim} H^{1}(C)$. The $Z$ distance $d_{Z}$ and the $X$ distance $d_{X}$ are the minimum Hamming weight of all nontrivial homology and cohomology classes, respectively. The distance $d$ is the minimum of $d_{X}$ and $d_{Z}$. Vice versa, a single chain complex can yield many CSS codes by taking any two consecutive boundary operators.

This is a fruitful perspective that allows us to import language and constructions from homological algebra into the theory of quantum codes. See Sec. VIII A for more details.

\section{Tanner graphs}

A linear binary code $C$ can be represented by a Tanner graph. A Tanner graph is a bipartite graph where each side of the partition corresponds to the bits and checks, respectively. Bits are connected to the checks in which they appear; see Fig. 1.

Analogously, a quantum CSS code can be described by a Tanner graph with three layers, representing $X$-checks,

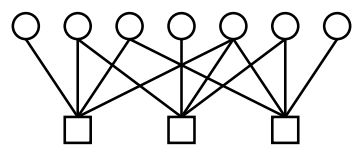

FIG. 1. The Tanner graph of the $[7,4,3]$ Hamming code. Physical bits are represented by circles and checks are represented by squares.

physical qubits, and Z-checks: see Fig. 2. A check acts on the qubits incident in the Tanner graph.

Accordingly, the incidence matrices between the layers are given by the parity-check matrices $H_{X}$ and $H_{Z}$, respectively. The commutativity constraint in Eq. (1) translates to the condition that the intersection of the neighborhoods of each $X$-check and $Z$-check contains an even number of physical qubits.

\section{Manifolds and cell complexes}

The toric code is arguably the most well-known CSS quantum code $[8,9]$. It is defined from a tessellation of a torus with square tiles, where edges correspond to physical qubits and the stabilizer checks correspond to faces ( $Z$-checks) and vertices ( $X$-checks). Each check acts on all its incident qubits or edges and the logical operators correspond to noncontractible loops.

Interesting quantum codes can be derived from tessellations of surfaces or manifolds other than the torus. For example, Shor's $[[9,1,3]]$ code can be constructed from a tessellation of the real projective plane, the nonorientable cousin of the torus; see Ref. [10]. In the same way as in the toric code, $X$-checks, physical qubits, and $Z$-checks of Shor's $[[9,1,3]]$ code are associated with vertices, edges, and faces of the tessellation, and checks act on their incident edges. This is shown in Fig. 3, where a tessellation of the projective plane with seven faces, nine edges, and three vertices is depicted. Pairs of antipodal points on the circle are identified. Four of the faces are adjacent to two edges and hence give rise to $Z$-checks of weight 2 . One face is adjacent to six edges, giving rise to a $Z$-check of weight 6. Similarly, all $X$-checks, which are associated with the

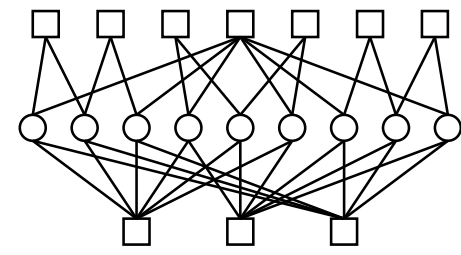

FIG. 2. The Tanner graph of the Shor code. Physical qubits are represented by circles, $X$-checks are represented the squares at the bottom, and $Z$-checks are represented by the squares at the top. We chose a linearly dependent set of stabilizer checks to establish a connection to a geometric interpretation of Shor's code; see Fig. 3. 


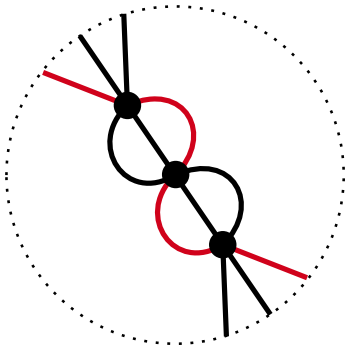

FIG. 3. The Shor code [7] as a tessellation of the real projective plane; see Ref. [10]. Antipodal points on the circle are identified. The code has three $X$-checks, nine physical qubits, and seven $Z$-checks represented by vertices, edges, and faces, respectively. The red line represents a logical $Z$-operator.

vertices, have weight 6 . This should be compared with Fig. 2, which represents the same code by a Tanner graph. From both representations, one can immediately extract the parity-check matrices $H_{X}$ and $H_{Z}$ as incidence matrices.

This construction generalizes to tessellations of other surfaces and to higher-dimensional manifolds $M$. We call the $i$-dimensional elements of the tessellation " $i$-cells," so vertices are 0 -cells, edges are 1-cells, faces are 2-cells, etc. Given a tessellation of a $D$-dimensional manifold, we identify $i$-cells of the tessellation $(0<i<D)$ with qubits, $X$-checks with $i-1$-cells, and $Z$ checks with $i+1$-cells. Nontrivial logical $Z$-operators correspond to subsets of $i$-cells that have no boundary and do not arise as the boundary of a subset of $i+1$-cells. The vector spaces spanned by $i$-cells together with the boundary operator form the socalled cellular chain complex; see Sec. 2.2 in Ref. [35]. This connects the homological perspective of Sec. II B 1 with the geometrical perspective. Moreover, there is also a relation to Tanner graphs by considering the Hasse diagram of the tessellation; see Sec. 2.2 in Ref. [36]. The logical $X$-operators are obtained in the same way by taking the dual tessellation or by considering cohomology classes.

The distance is related to the $i$-systole $\operatorname{sys}_{i}(M)$ of $M$, which is the length, area, or volume of the smallest noncontractible $i$-dimensional submanifolds of $M$. This yields families of codes by taking finer and finer tessellations. For reasonable tessellations, these families are LDPC codes.

\section{GEOMETRICAL CONSTRUCTIONS}

Arguably the most famous quantum codes are the toric code and its planar variant the surface code. These two examples are part of a much larger family of codes derived from geometrical objects; see Sec. II B 3. Importantly, the properties of these codes are determined by the geometry of the underlying space, so tools from geometry become amenable to quantum error correction.

\section{A. Hyperbolic quantum codes}

Quantum codes with a finite encoding rate $k / n \rightarrow R>0$ for $n \rightarrow \infty$ naturally arise from manifolds of negative curvature, called "hyperbolic manifolds." The reason for this lies in the Gauß-Bonnet-Chern theorem, which relates the geometry of a manifold to its topology [37]. More concretely, it shows that for hyperbolic manifolds of even dimension $D=2 i$, the dimension of the homology group $H_{i}$ grows linearly with the total volume of the manifold. Hence, the associated quantum code has a linear encoding rate. Therefore, any code derived from such a manifold (see Sec. II B 3) will have a constant encoding rate.

Compare this with the $L \times L$ toric code, where the logical operators of minimum weight correspond to onedimensional submanifolds (circles). The number of these is two, regardless of $L$. The same is true for higher dimensions; for example, one can define a four-dimensional (4D) toric code [27] where the logical operators of minimum weight correspond to six two-dimensional (2D) tori.

\section{Hyperbolic surface codes}

Hyperbolic surface codes are the closest relatives of the toric code. They are defined in exactly the same way as the toric code, except that the tessellations are derived from hyperbolic geometry.

If we consider a closed surface with a hyperbolic metric, then the Gauß-Bonnet-Chern theorem [37] mentioned above can be used to derive an exact formula for the number of encoded logical qubits. In particular, for regular tessellations based on regular polygons with $r$ sides and $s$ polygons meeting at each edge, one can show that the number of logical qubits is given by $k=(1-2 / r-2 / s) n+2$. See Figure 4 for an example of a hyperbolic surface code derived from a surface of genus 3 . The stabilizer check weight is $r$ for $Z$-checks and $s$ for $X$-checks, so there is a
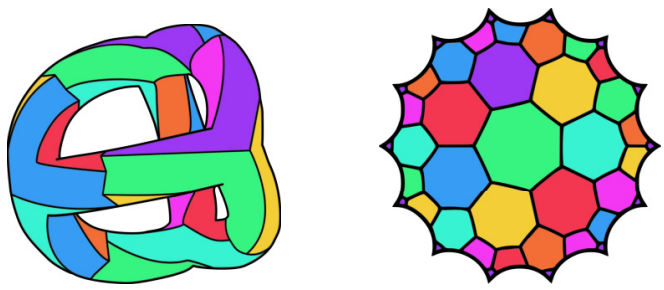

FIG. 4. A hyperbolic surface of genus 3 tessellated by heptagons. It gives rise to a code with parameters $n=84, k=6$, $d_{X}=4$, and $d_{Z}=8$. The colors have no intrinsic meaning and are included only as a guide for the eye. A weight- $4 X$-operator goes through the following four faces on the right: magenta (top), violet, green (middle), yellow (below), and back to the same magenta face (periodic boundary). A weight- $8 Z$-operator runs along the left-hand side of these faces. 
trade-off between check weight and encoding rate. Hyperbolic surface codes exist with check weights of 5 and 4 for $X$-checks and $Z$-checks, or vice versa $[38,39]$. Hyperbolic surface codes and their properties were discussed in Refs. [40-42]. A general construction as well as a planar version were introduced in Ref. [38]. Delfosse and Zémor [23] used hyperbolic surface codes to obtain results in percolation theory.

The distance of hyperbolic surface codes is logarithmic, which suffices to prove that a threshold under minimumweight decoding exists [43]. Many decoders that apply to the surface code can be used directly for hyperbolic surface codes, such as minimum-weight perfect matching [38] and the union-find decoder [44]. However, this means that error suppression on the logical qubits for physical error rates below the threshold scales only polynomially with the system size. Nevertheless, numerical simulations show that hyperbolic surface codes offer a reduction of physical qubits in the phenomenological noise model $[38,39]$ and gate-based noise model [45]. On the basis of the symmetry of hyperbolic surface codes, it is possible to find optimal measurement schedules of the check operators [45], and they are currently the only finite-rate quantum codes for which such schedules are known.

Higgott and Breuckmann [45] showed that hyperbolic surface codes can be turned into subsystem codes with weight-3 checks. There also exist hyperbolic versions of color codes $[42,46,47]$ that could simplify the implementation of logical gates.

\section{Higher-dimensional hyperbolic codes}

Lubotzky and Guth [48] showed that codes derived from hyperbolic manifolds of dimension larger than 2 give quantum codes with distance scaling as $d \in \Theta\left(n^{\alpha}\right)$ for some $\alpha>0$. They constructed families of 4D hyperbolic quantum codes such that $\alpha>0.1$. For arithmetic 4D hyperbolic manifolds, they established an upper bound of $\alpha<0.3$. However, it is an open problem whether these bounds hold for quantum codes derived from general 4D hyperbolic manifolds.

Hastings [49] proposed an efficient local decoding strategy for 4D hyperbolic codes. However, despite having a distance scaling like $n^{\alpha}$, Hasting's decoder is shown to correct errors only up to size $\log n$.

The description of the codes by Lubotzky and Guth [48] is implicit. Londe and Leverrier [50] considered a tessellation of 4D hyperbolic space by hypercubes giving rise to a family of codes with asymptotic encoding rate $R \geq 17 / 360$. A construction based on a self-dual tessellation by 120 -cells was given by Breuckmann and Londe [51], giving an asymptotic encoding rate $R \geq 13 / 72$. Furthermore, they showed how topological coverings can be used to reduce the size of these codes and performed simulations of the codes using a belief-propagation (BP) decoder that indicate that it has intrinsic robustness against measurement errors; see Sec. VIC.

\section{B. Freedman-Meyer-Luo codes}

Hyperbolic geometry was used in earlier work by Freedman et al. [52] to construct a family of quantum codes with parameters $[[n, 2, \Omega(\sqrt[4]{\log n} \sqrt{n})]]$ [53]. These codes held the record for distance scaling for around 20 years, until the record was broken in 2020 by several studies discussed in Sec. IV. The arguments used in Ref. [52] are quite involved and beyond the scope of this Perspective. However, we sketch the main ideas behind the construction to give the intuition behind the distance scaling for the interested reader.

We now sketch the construction of the underlying manifolds to give intuition for the distance bound: First, take a closed hyperbolic surface $\Sigma_{g}$ of genus $g$ and take the Cartesian product with the interval $[0,1]$ of unit length. The length of the shortest noncontractible loop on $\Sigma_{g}$ is called " 1 -systole" and is denoted by $\operatorname{sys}_{1}\left(\Sigma_{g}\right)$. We identify the two ends of $\Sigma_{g} \times[0,1]$ with a twist of length $\sqrt{\operatorname{sys}_{1}\left(\Sigma_{g}\right)}$, so the 1 -systole of the resulting threedimensional manifold is $\sqrt{\operatorname{sys}_{1}\left(\Sigma_{g}\right)}$; see Fig. 5. All noncontractible loops of length $\operatorname{sys}_{1}\left(\Sigma_{g}\right)$ coming from $\Sigma_{g}$ are removed by surgery, so we obtain a 3-manifold that we denote by $P_{g}$. Since the interval $[0,1]$ has unit length, the three-dimensional volume of $P_{g}$ is proportional to the area of $\Sigma_{g}$. Furthermore, because of the Gauß-BonnetChern theorem (see Sec. III A) we have area $\left(\Sigma_{g}\right)=\Theta(g)$. The final step in the construction of the manifolds is to take a loop $S^{1}$ of length $g / \sqrt{\log g}$ and take the Cartesian product with $P_{g}$. The resulting 4-manifold then has four-dimensional volume $\operatorname{vol}_{4}\left(P_{g} \times S^{1}\right)=g^{2} / \sqrt{\log g}$.

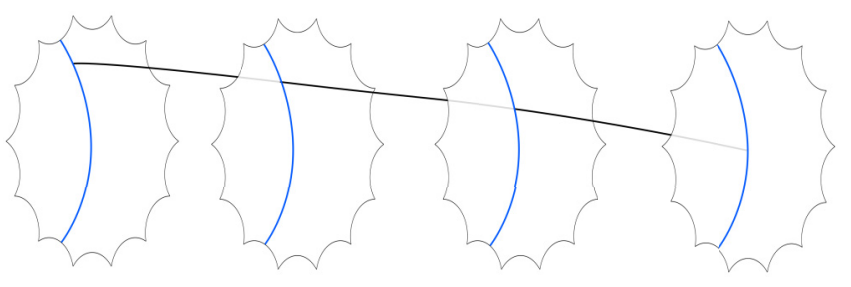

FIG. 5. Illustration of a crucial step in the construction of Freedman-Meyer-Luo codes. The hyperbolic polygon is the fundamental domain of a hyperbolic surface $\Sigma_{g}$ with genus $g$ (see Fig. 4). The blue line corresponds to a geodesic on the surface of length $\Theta(\log g)$. All points on the surface are translated by a distance $\Theta(\sqrt{\log g})$ along this geodesic. The thick black line traces out the position of a single point in the product $\Sigma_{g} \times[0,1]$. The two ends of the product, corresponding to coordinates 0 and 1 in the interval, are then identified so that we obtain $\Sigma_{g} \times S^{1}$ with a twist. 
By tessellating the manifolds uniformly, we obtain a cell complex on which we can define a homological quantum code by identifying the 2-cells (faces) with qubits, 1-cells (edges) with $X$-checks, and 3-cells (volumes) with $Z$-checks, as described in Sec. II B 3. The physical qubits derived from these manifolds have the number of qubits $n$ scaling with $\operatorname{vol}_{4}\left(P_{g} \times S^{1}\right)$. The logical operators correspond to noncontractible surfaces inside the 4-manifold. Their area is called the "2-systole" and they have size sys $_{2}\left(P_{g} \times S^{1}\right)=\Theta(g)$, leading to the distance bound. See Ref. [54] for a review of the construction that covers all the details.

\section{Haah's code}

Haah developed a general formalism that describes translation-invariant stabilizer codes that are local in $D$ dimensional Euclidean space [55]. Arguably the most famous example of a quantum code constructed by Haah's method is Haah's cubic code, which is defined on a threedimensional cubic lattice of size $L \times L \times L$ with periodic boundary conditions and two qubits per site [56]. The number of encoded qubits in Haah's cubic code grows with $L$ and hence with the number of physical qubits. However, the exact number depends in a nontrivial way on $L$. For the distance of Haah's cubic code, only the bounds $\Omega(\sqrt[3]{n}) \leq d \leq O\left(n^{2 / 3}\right)$ are known [56,57]. An interesting feature of Haah's cubic code is that its logical operators are fractals. Although we classify Haah's code as a geometric code here, as it is defined on a cubic lattice, it can also be understood as a special case of a product construction discussed in Sec. IV.

Open problem. What is the asymptotic distance scaling of Haah's cubic code?

Haah's code is a candidate for a self-correcting quantum memory. By defining a local Hamiltonian that has the parity checks as energy-penalty terms, we obtain a physical system with the quantum code as its ground state. A self-correcting memory would be such a system that is inherently robust with regard to thermal noise, without the need for an active decoding procedure. As this is not the focus of this text, we refer the reader to Ref. [58] for more background.

\section{Bounds on parameters}

While geometry is a useful tool for the construction of quantum LDPC codes, it also comes with restrictions. Fetaya [59] showed that any code derived from the tessellation of a surface, either closed or with a boundary, must have its distance bounded as $d^{2} \leq O(n)$. Delfosse [42] extended Fetaya's result to the bound $k d^{2} \leq O\left(\log ^{2}(k) n\right)$.

For higher dimensions, Bravyi et al. [60] showed that for any $[[n, k, d]]$ stabilizer code on a $D$-dimensional
Euclidean lattice, $k d^{\alpha} \leq O(n)$, where $\alpha=2 /(D-1)$. The Bravyi-Poulin-Terhal bound does not extend to nonEuclidean lattices, as for $D=2$ the bound is violated by hyperbolic surface codes (see Sec. III A 1). A class of codes conjectured to satisfy the Bravyi-Poulin-Terhal bound in three dimensions was introduced by Devakul and Williamson [61].

Open problem. Can the above bounds be modified or extended to non-Euclidean lattices in higher dimensions?

This problem seems challenging as it relates to deep questions in a subfield of mathematics called "systolic geometry," which analyzes the volume scaling of noncontractible submanifolds [62].

\section{PRODUCT CONSTRUCTIONS}

Classical coding theory is a long-established field, and it would be desirable to transfer results into quantum coding theory. In this section we describe various product constructions that allow us to build quantum codes from classical codes and/or quantum codes. These constructions are at the heart of recent breakthrough results in the theory of quantum LDPC codes.

The first class of examples are incarnations of the tensor product of chain complexes from homological algebra. There are the hypergraph product codes by Tillich and Zémor [63] and the homological product codes of Hastings and Bravyi [64], constructing a quantum code from two classical codes. The distance balancing of Hastings [65] and Evra et al. [66] is achieved by taking tensor products of quantum codes with classical codes, while the codes of Kaufman and Tessler [67] use iterated tensor products of quantum codes.

There are multiple improvements and generalizations of these product constructions. Hastings et al. [68] defined fiber bundle codes that introduce a twist in the tensor product so as to increase the distance. Another approach is found in the generalized hypergraph product and lifted product of Panteleev and Kalachev [57,69] as well as the balanced product of Breuckmann and Eberhardt [70]. All these are very closely related to each other; see Sec. VIII A. In 2020, the distance record of Freedman et al. from 2002 (see Sec. III B) was broken multiple times by use of these product constructions. These are breakthrough results, as they surpass the $\sqrt{n}$ polylog $n$ distance barrier which was by some believed to be unsurpassable. While many of the constructions do not have a constant encoding rate and hence do not satisfy the assumptions of Gottesman's constant overhead theorem [18], they constitute an important step in the pursuit of good quantum LDPC codes. Independently of asymptotic results, these constructions provide tools to construct concrete examples of codes worth studying, as done in Ref. [69]. 


\section{A. Tensor (hypergraph) products}

The tensor product of vector spaces extends to the notion of the tensor product of chain complexes. This is a classical construction in homological algebra closely related to the Cartesian product of topological spaces; see Sec. 2.7 in Ref. [71].

In particular, new quantum codes can be constructed by tensor products of classical codes and/or quantum codes using tensor products.

The first product construction in this spirit is the hypergraph product introduced by Tillich and Zémor [63] in 2009. The hypergraph product constructs a [ $\left[n_{1} n_{2}+\right.$ $\left.\left.r_{1} r_{2}, k_{1} k_{2}, \min \left\{d_{1}, d_{2}\right\}\right]\right]$ quantum code from two classical $\left[n_{i}, k_{i}, d_{i}\right]$ codes with $r_{i}$ linearly independent checks for $i=1,2$. Its stabilizer checks are a combination of the physical bits and parity checks of the classical codes; see Fig. 6. By taking the hypergraph product of suitable classical LDPC codes, Tillich and Zémor achieved quantum LDPC codes with a constant encoding rate and distance $d \in \Theta(\sqrt{n})$. Hypergraph products were used to define quantum expander codes (see Refs. [72,73]) using Sipser-Spielman expander codes [74]. Furthermore, there are constant factor improvements [75] and higher-dimensional generalizations [76] of hypergraph products.

While Tillich and Zémor's definition is of combinatorial nature and uses Tanner graphs (see Sec. II B 2), it is equivalent to taking the tensor product of two chain complexes induced by the classical codes; see Sec. II B 1. This perspective was, for example, taken by Hasting and Bravyi [64] in 2011 with their homological product codes and by Audoux and Couvreur [77] in their work on tensor products of CSS codes. The tensor product has the advantage

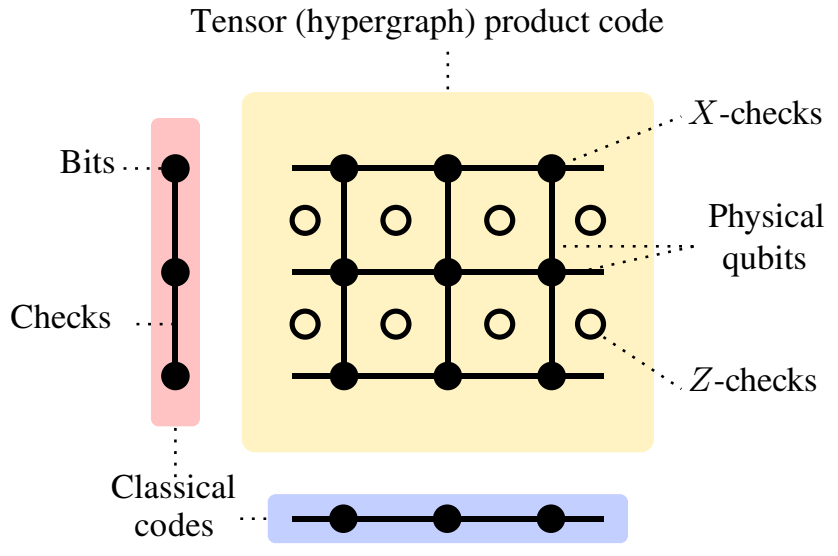

FIG. 6. The tensor (hypergraph) product of two repetition codes yields a surface code. The physical qubits of the quantum code are represented by edges and correspond to pairs of bits (vertical) or pairs of checks (horizontal) in the two repetition codes. In the hypergraph product construction, one of the classical codes is transposed, which is not depicted here. over hypergraph products of being defined for arbitrary chain complexes and not just classical codes, which gives immediate higher-dimensional generalizations. For more details, see Sec. VIII A c.

In April 2020, generalizing a construction of Hastings [65], Evra et al. [66] introduced a distance-balancing procedure for quantum codes using tensor products. They showed that the tensor product of a $\left[\left[n, k, d_{X}, d_{Z}\right]\right]$ quantum code with $r_{X} X$-checks and a classical $[m, l, d]$ code with $r$ checks yields a $\left[\left[n m+r_{X} r, k l, d_{X}, d_{Z} d\right]\right]$ quantum code. Armed with this new tool, Evra et al. broke the distance record of Freedman et al. (see Sec. III B). Evra et al. considered Ramanujan complexes, a higher-dimensional generalization of Ramanujan graphs; see Ref. [78]. Using Ramanujan complexes directly would yield quantum codes with distances $d_{X} \in \Theta(\log n)$ and $d_{Z} \in \Theta(n)$. By applying distance balancing to these, Evra et al. constructed codes with distance $d \in O(\sqrt{n} \log n)$.

Just 4 months later, in August 2020, Kaufman and Tessler [67] set a new record with $d \in O\left(\sqrt{n} \log ^{m}(n)\right)$ for arbitrary positive integers $m$ by using iterated tensor products of Ramanujan complexes.

\section{B. Fiber bundles}

One month after Kaufman and Tessler's results [67], in September 2020, the $\sqrt{n}$ polylog $n$ distance barrier was broken by the fiber bundle codes of Hastings et al. [68], a generalization of the tensor product.

Much like tensor products, fiber bundle codes are constructed from two classical codes, referred to as the "base code" and the "fiber code." While the number of physical qubits, logical qubits, and checks is the same as in the tensor product, certain twists are introduced in the checks of the fiber, permuting the position of the qubits in the direction of the fiber. The twists are determined by a collection of automorphisms of the fiber code that are specified for every pair of bit and incident check of the base code. This can result in an increased distance of the resulting code. A trivial example is the twisted toric code, where the lattice is displaced along the vertical direction. Because of the twist, the nontrivial horizontal loop representing a logical $Z$-operator has to take a "detour" to close up on itself.

The concept is derived from the topological notion of fiber bundles [37], and fiber bundle codes can be visualized as the fiber code varying nontrivially over the base code (see Fig. 7), much like the Möbius strip, where the unit interval (fiber) varies nontrivially along the circle (base), or in the Klein bottle, where a circle (fiber) varies over another circle (base). For more details, see Sec. VIII A d.

Hastings et al. applied the fiber bundle construction to a random classical code as the base, a cyclic repetition code as the fiber, and a random choice of twists. By homological and probabilistic arguments, they showed that this yields families of quantum LDPC codes with 


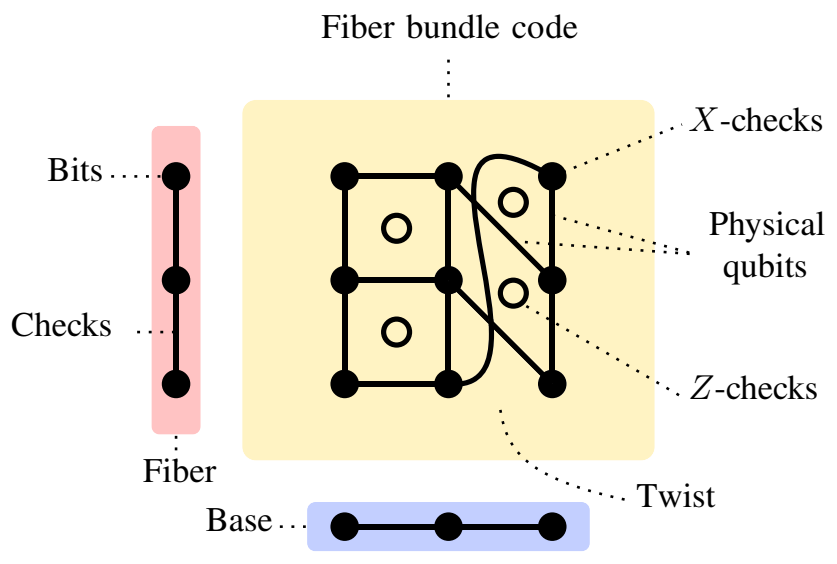

FIG. 7. A fiber bundle code obtained from two repetition codes. Here we assume periodic boundary conditions in the vertical direction.

$k \in \Theta\left(n^{3 / 5} /\right.$ polylog $\left.n\right)$ logical qubits and distance $d \in$ $\Omega\left(n^{3 / 5} /\right.$ polylog $\left.n\right)$, a big step in the endeavor toward good quantum LDPC codes.

\section{Lifted products}

Panteleev and Kalachev [57] broke the record of Hastings et al. just 2 months later, in December 2020, using a different improvement of tensor product codes. In 2019 [69] they had introduced generalized hypergraph product codes, which they later renamed as lifted product codes. The lifted product construction allows one to decrease the number of physical qubits in the tensor product by a reduction of symmetry.

Specifically, the construction takes as input two classical codes given by their parity-check matrices. It assumes that the matrices admit a block decomposition into pairwise commuting submatrices of size $\ell \times \ell$ for some $\ell$. First, the hypergraph/tensor of the classical codes is taken. Then the parity-check matrices of the resulting quantum code inherit a natural block decomposition into submatrices of size $\ell^{2} \times \ell^{2}$. Next, the lifted product is obtained by collapsing each of these submatrices of size $\ell^{2} \times \ell^{2}$ to matrices of size $\ell \times \ell$ via summation over all of their $\ell \times \ell$-sized blocks. The pairwise commutativity assumption ensures that the result defines a CSS code.

The lifted product reduces the number of physical qubits by a factor of $\ell$ in comparison with the hypergraph/tensor product. See Appendix A e for more details.

Panteleev and Kalachev [57] studied a special case of lifted product codes constructed from coverings of SipserSpielman expander codes; see Ref. [74]. Their quantum codes are constructed from an $s$-regular expander graph (the base), a cyclic covering of this graph of degree $\ell$ (the lift), and a classical local code on $s$ bits. By the grouping together of vertices and edges in the lift graph that map to the same vertex or edge in the base graph, the incidence matrix of the lift graph has a natural block decomposition into submatrices of size $\ell \times \ell$. The submatrices all commute since they correspond to a cyclic permutation. Next, the lift graph is combined with the local code, using the construction of Sipser and Spielman. The resulting paritycheck matrix inherits a block decomposition. In the last step, the lifted product of this matrix with the adjacency matrix of the cycle graph of size $\ell$ (the parity-check matrix of cyclic repetition code) is formed.

Most remarkably, they establish tight distance bounds for these lifted product codes assuming (co)expansion properties of the associated classical expander code. By a random choice of graph, cover, and local code, they construct quantum LDPC codes with logical bits in $\Theta\left(n^{\alpha} \log n\right)$ and distance in $\Omega\left(n^{1-\alpha / 2} \log n\right)$ for any $0 \leq$ $\alpha<1$. Thereby they achieve quantum LDPC codes with almost linear distance.

\section{Balanced products}

Almost simultaneously to Panteleev and Kalachev's work, Breuckmann and Eberhardt [70] introduced balanced product codes. Similarly to lifted products, the balanced product construction is based on a reduction of symmetry in the hypergraph/tensor product. The balanced product is defined for two classical codes with a common symmetry group and arises by modding out the action of the group on their hypergraph/tensor product. The concept is derived from the balanced product of topological spaces, a classical construction in topology that is commonly used to construct fiber bundles from principal bundles [37].

We now explain the construction in more detail. To begin, assume we are given a classical code with a group $H$ acting on the bits and checks via permutation such that the incidence relation between bits and checks is preserved. One can form the quotient code by identifying bits and checks that lie in the same orbit of the action, thereby reducing the length of the code. Now, given two classical codes $C$ and $D$ on which a group $H$ acts, one can form the balanced product $C \otimes_{H} D$ as follows. First, the tensor (hypergraph) product code $C \otimes D$ is formed. There is an induced action of $H$ on the physical qubits and checks of this quantum code. The balanced product $C \otimes_{H} D$ is obtained by identifying physical qubits and checks in the same orbit, much like in the classical quotient code. The balanced product construction also has greater generality. For example, one can form the balanced product of two quantum codes or chain complexes. See Sec. VIII A f for more details.

In Fig. 8 the balanced product of two repetition codes (cycle) graphs of sizes 3 and 6 is visualized. Here $\mathbb{Z}_{3}$, the cyclic group of order 3 , acts on both codes via rotation by $120^{\circ}$. Their hypergraph/tensor product is a $3 \times 6$ toric code on which the group $\mathbb{Z}_{3}$ acts via rotating the torus in both 


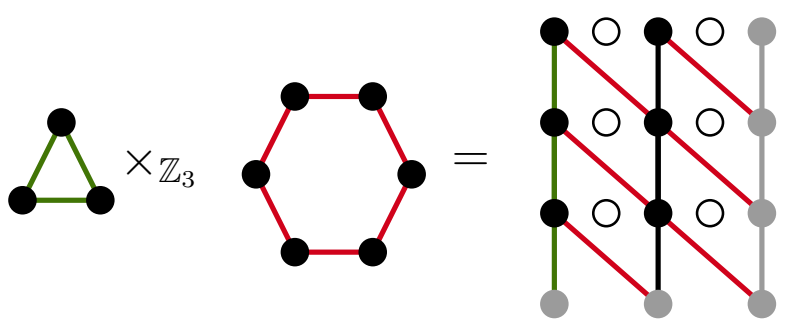

FIG. 8. The balanced product of the length- 3 and length- 6 cyclic graphs over $\mathbb{Z}_{3}$. This gives a $3 \times 2$ twisted toric code, which is a $[[12,2,3]]$ quantum code. Gray edges and vertices are included only to visualize the periodic boundaries. This code has an equivalent interpretation as a fiber bundle with a length-2 cycle graph as the base and a length-3 cycle graph as the fiber. It is also a lifted product of the boundary operators $\partial_{1}$ of the two cycle graphs.

directions by $120^{\circ}$ simultaneously. Now all $X$-checks (vertices), physical qubits (edges) and $Z$-checks (faces) that lie in the same orbit of the action are identified. The resulting balanced product code is a $3 \times 2$ twisted toric code with parameters [[12, 2, 3]].

Breuckmann and Eberhardt applied the balanced product to Sipser-Spielman codes and repetition codes with cyclic symmetry. The Sipser-Spielman codes are derived from Lubotzky-Phillips-Sarnak expander graphs, which are Cayley graphs of projective linear groups over a finite field PSL $(2, q)$; see Ref. [79]. The resulting codes are nonrandom and achieve a number of logical qubits $k \in$ $\Theta\left(n^{4 / 5}\right)$ and distance $d \in \Omega\left(n^{3 / 5}\right)$.

\section{E. The possibility of good quantum LDPC codes}

The fiber bundles, lifted products and balanced products open to the door to many new code families. They give hope that it possible to construct good quantum LDPC codes, and so codes with a constant rate and linear distance. However, we believe that it is not sufficient to consider only cyclic (or more generally Abelian) twists or symmetries, such as those used in the previous three sections. This is supported by arguments of Panteleev and Kalachev [57], (Sec. IV C), who showed that their bounds $k \in \Theta\left(n^{\alpha} \log n\right)$ and $d \in \Omega\left(n^{1-\alpha / 2} \log n\right)$ for $0 \leq \alpha<1$ are in some sense optimal in these cases.

One promising idea to circumvent these bounds is to take non-Abelian twists. This is possible in the balanced product construction by taking a non-Abelian group $H$. For example, one could take the balanced product of two copies of a Sipser-Spielman code derived from a Cayley graph of a non-Abelian group such as $\operatorname{PSL}(2, q)$. While one can show that this yields codes with a linear rate, it is not yet clear how to determine their distance.
Open problem. Can the balanced product of two expander codes derived from Cayley graphs be used to construct good LDPC codes [70], (Sec. VI)?

Balanced product codes have the advantage of being symmetric in their two input factors. The construction can easily be used to construct codes that are isomorphic to their dual, hence rendering any distance balancing unnecessary.

\section{F. $X Y Z$ products}

Leverrier et al. [80] considered $X Y Z$ product codes, a product construction of stabilizer codes that are not CSS codes. The idea was suggested in Ref. [81] and generalizes a three-dimensional non-CSS code due to Chamon $[82,83]$. An $X Y Z$ product code is defined by a tensor product of three classical codes. Each check contains Pauli $X$, $Y$, and $Z$ operators. Leverrier et al. argue that the distance of $X Y Z$ product codes could be as high as $\Theta\left(n^{2 / 3}\right)$ as logical operators have natural representations as " $2 \mathrm{D}$ objects" in the product. However, there are no known general lower bounds on the distance. Bravyi et al. [83] showed that for the Chamon code, which is the $X Y Z$ product of three repetition codes with block lengths $n_{1}, n_{2}$, and $n_{3}$, the number of encoded qubits is given by $4 \operatorname{gcd}\left(n_{1}, n_{2}, n_{3}\right)$. Recently, a $2 \mathrm{D}$ version of the Chamon code was found to perform remarkably well in numerical simulations when the noise is biased [84].

Open problem. What is the minimum distance and performance of quantum XYZ product codes?

\section{OTHER CONSTRUCTIONS RELATED TO LDPC CODES}

In this section we describe other families of quantum codes that are not LDPC codes under our strict definition; see Sec. II A 2. However, they are sometimes called "LDPC codes" in the literature and are based on very interesting ideas, which is why we include them here.

\section{A. Bravyi-Hastings codes}

Bravyi and Hastings [64] applied the tensor product construction (see Sec. IV A) to two random, non-LDPC CSS codes with check weights $\Theta(n)$. They showed that with high probability the resulting codes have parameters $[[n, \Theta(n), \Theta(n)]]$ (i.e., they are good codes). However, the codes are not LDPC codes as the check weights are in $\Theta(\sqrt{n})$. This improves on the earlier result obtained by Calderbank and Shor [13], who constructed good quantum codes with check weight $\Theta(n)$. The square root of the check weight comes from the fact that Bravyi and Hastings take the product of two codes with a linear check 
weight and that the check weights are additive in the product. This immediately suggests that the weight could be further suppressed by taking iterated products.

A related construction due to Hastings [85], under the assumption of a conjecture in geometry, achieves distance $d \in \Omega\left(n^{1-\epsilon}\right)$ for arbitrary $\epsilon>0$ and with logarithmic stabilizer weight.

Open problem. Does the iterated product of random codes provide a code family of good codes with stabilizer check weight scaling that is arbitrarily low?

\section{B. Bravyi-Bacon-Shor codes}

Bravyi-Bacon-Shor codes are generalizations of BaconShor subsystem codes defined in Ref. [86] and studied by Yoder $[87,88]$. They are defined from a binary matrix $A \in \mathbb{F}_{2}^{m_{1} \times m_{2}}$ by placing physical qubits on an $m_{1} \times m_{2}$ square grid with a physical qubit placed at position $(i, j)$ if and only if $A_{i, j}=1$. The gauge operators are generated by $X X$ interactions between any two consecutive qubits sharing a column and $Z Z$ interactions between any two consecutive qubits sharing a row. The number of physical qubits $n$ of the resulting code is the number of nonzero entries in $A$. Bravyi [86] furthermore showed that the number of logical qubits is $k=\operatorname{rk} A$ and that the minimum distance is the minimum Hamming weight of the row span and column span of $A$; that is, $d=\min _{c \in V}|c|$, where $V=\left(\operatorname{im} A \cup \operatorname{im} A^{T}\right) \backslash\{0\}$.

Yoder [87] considered taking two classical codes with parameters $\left[n_{1}, k, d_{1}\right]$ and $\left[n_{2}, k, d_{2}\right]$ with generating matrices $G_{1}$ and $G_{2}$ to define a Bravyi-Bacon-Shor code based on the matrix $A=G_{1}^{T} Q G_{2}$, where $Q \in \mathbb{F}_{2}^{k \times k}$ is any full-rank matrix. The resulting code then has between $\min \left\{n_{1} d_{2}, d_{1} n_{2}\right\}$ and $n_{1} n_{2}$ physical qubits, $k$ logical qubits, and distance $\min \left\{d_{1}, d_{2}\right\}$. In particular, when the classical input codes have a constant rate and a linear distance, then the resulting Bravyi-Bacon-Shor codes have optimal scaling for 2D subsystem codes. Furthermore, the resulting code inherits a decoder from the classical codes used for the construction.

Open problem. Can the Bravyi-Bacon-Shor codes be extended to the novel product constructions discussed in Ref. IV?

\section{Subsystem codes from quantum circuits}

Bacon et al. [89] showed that it is possible to obtain quantum codes such that each physical check has weight $O$ (1) with distance $\Theta\left(n^{1-\epsilon}\right)$, where $\epsilon \in O(1 / \sqrt{\log n})$. Furthermore, restricting the code to be spatially local in $D$ dimensional Euclidean space, they showed that a distance of $\Theta\left(n^{1-\epsilon-1 / D}\right)$ can be obtained. The physical checks correspond to gauge operators and not to the actual stabilizer checks. The stabilizer checks can be written as products of the gauge operators, so the outcome of stabilizer measurements can in principle be inferred from the measurements of the gauge operators. The number of gauge factors of a stabilizer is not bounded; in other words, the actual stabilizer checks have unbounded weight, so this code family is not a family of LDPC codes.

We briefly sketch the main idea behind the construction. The authors show that a quantum circuit can be mapped onto a quantum code by associating the gates with gauge operators that act on physical qubits positioned between the gates. It can then be shown that if the mapping is applied to a suitable error-detection circuit of a stabilizer code, then the resulting subsystem code has the same logical operators up to multiplication with gauge operators. The actual parameters stated earlier can be obtained by taking a quantum code with parameters $\left[\left[n_{0}, 1, \Theta\left(n_{0}\right)\right]\right]$, which is guaranteed to exist by Ref. [90], and concatenate it with itself a suitable number of times.

In this construction the distribution of the stabilizer check weights is nonuniform but is logarithmically distributed. Although it is unlikely that these codes can have a threshold, it might still be worthwhile to find an efficient decoder to test whether the error suppression is competitive for relevant system sizes.

Open problem. Can the codes of Bacon et al. [89] be efficiently decoded?

\section{Approximate codes from space-time circuit Hamiltonians}

An interesting approach was taken by Bohdanowicz et al. [91]. Similarly to the codes of Bacon et al. discussed in Sec. VC, they derived quantum codes from quantum circuits. The parameters of their code are $[[n, \Omega(n / \operatorname{polyl} \log n), \Omega(n /$ polylog $n)]]$. They define their code as the ground space of a local Hamiltonian, where each term operates on nine qubits and each qubit participates in polylog $n$ many terms. The codes are nonstabilizer codes (i.e., the terms of the Hamiltonian are not given by Pauli operators), so many fault tolerance techniques developed for stabilizer codes do not apply. For example, it is not clear how to measure the energy of each term of the Hamiltonian or how to process the information for a recovery. Furthermore, the codes are approximate codes, which means that the fidelity of the encoded state after a recovery is only $1-\epsilon$, where $\epsilon \in o(1)$.

Their construction uses encoding circuits of good quantum codes of polylogarithmic depth, which are guaranteed by Ref. [92]. This encoding circuit is mapped onto a local Hamiltonian that contains the valid computations of the circuit in its ground space [93] and has a spectral gap that scales as $\Omega\left(1 / n^{\alpha}\right)$ for some $\alpha>0$. They show that for arbitrary errors a recovery operation exists that restores the initial state with high fidelity. 
A different approach to nonstabilizer codes was taken by Movassagh and Ouyang [94], who demonstrated how to map classical codes into the ground space of quantum spin chain Hamiltonians.

Open problem. Can nonstabilizer codes and approximate codes give rise to practical and competitive fault tolerance schemes?

\section{CHALLENGES AND OPPORTUNITIES}

\section{A. Reduction in overhead}

A major achievement of fault-tolerant quantum computing is the threshold theorem $[8,19,20]$, which shows that fault-tolerant quantum computation is possible with polylogarithmic overhead of physical qubits in the length of the computation. A theorem due to Gottesman [18] shows that it is even possible to perform quantum computation with only constant overhead in resources.

More precisely, Gottesman shows the following: assuming we have a family of LDPC codes with parameters $\left[\left[n_{i}, k_{i}, d_{i}\right]\right]$ such that (a) it has a constant rate $\liminf _{i \rightarrow \infty} n_{i} / k_{i}=R>0$, (b) its elements are polynomially spaced (i.e., $0<n_{i}-n_{i-1}<n_{i}^{\beta}$ for some constant $\beta>0$ ), and (c) there exists an efficient decoding algorithm which for suitably low noise parameters suppresses errors as $1 / g\left(n_{i}\right)$ for $i \rightarrow \infty$, where $g$ is some nondecreasing function, then any suitably large quantum circuit on $k$ qubits can be approximated with arbitrary precision if the noise of the components is below a certain threshold parameter using at most $\eta k / R$ physical qubits, where $\eta>1$ controls the threshold. In particular, this result gives an exact upper bound compared with the earlier threshold theorems, which can have very large constants hidden in the asymptotic analysis [95].

Fawzi et al. [96] showed that the assumptions of Gottesman's theorem can be satisfied using a hypergraph product code built from expander codes [97] decoded by a simple decoder that they call the "small-set-flip decoder." It is very likely that other codes discussed in Secs. III and IV could fulfill the requirements of Gottesman's theorem as well. The key to this is finding decoders that are sufficiently simple so as to be able to prove the required error suppression.

Open problem. Which quantum LDPC codes can be used for Gottesman's constant overhead theorem?

\section{B. Logical operations}

Gottesman's theorem guarantees a constant overhead by performing operations sequentially with logical gates implemented using ancilla states. However, it does require a minimum number of logical qubits to become effective, and this number has yet to be determined. Hence, there may be schemes that could potentially turn out to be more practical. For an overview of the leading proposals of implementing operations on codes not discussed here, see Ref. [98].

Bravyi and König [99] showed that there is a tradeoff between the implementability of constant-depth logical gates and the spacial locality in Euclidean space; see also Ref. [100]. A corollary of their result is that any code that is spatially local in two dimensions can only have constant-depth logical gates belonging to the Clifford group. Therefore, to implement logical gates in codes such as the surface code or two-dimensional color codes, we need to execute circuits of depth scaling with the code size.

One could therefore expect that LDPC codes that are not bound by locality might offer an advantage. Not much is known regarding logical gates for general LDPC codes.

Code deformations were considered for hyperbolic surface codes to perform controlled NOT gates [39]. Krishna and Poulin [101] considered generalizations of code deformation techniques of the surface code to hypergraph product codes (see Sec. IV A) to implement Clifford gates. On the other hand, Burton and Browne [102] showed that it is not possible to obtain logical gates with circuits of depth 1 (transversal gates) outside the Clifford group using hypergraph product codes.

A different approach was taken by Jochym and O'Connor [103], who showed that by taking the tensor product of two suitable quantum codes with complementary sets of gates it is possible to perform the logical operations of either and thus obtain a fault-tolerant and universal set of gates. Such a scheme may be an alternative to Gottesman's protocol [18], which achieves universality using ancillary states to obtain constant overhead.

\section{Decoding}

For a general stabilizer code, it was shown by Iyer and Poulin [104] that optimal decoding (i.e., maximizing the success probability of reversing the error) is No. P-complete. However, it is often sufficient to consider a suboptimal decoding algorithm, such as minimum-weight perfect matching for the surface code [27].

Current decoding algorithms for the surface code or 2D topological codes suffer from a large time complexity, although progress has been made in reducing the time complexity of decoding the surface code $[44,105]$. Here, LDPC codes could offer an advantage. First, the time complexity of decoding algorithms often depends on the number of physical bits. LDPC codes can achieve higher encoding rates, offering the same level of protection, and consequently admit faster decoding. For example, applying minimum-weight perfect matching to hyperbolic surface codes can yield significant performance improvements in comparison with 2D surface codes. Second, LDPC codes offer simplified decoding algorithms, significantly 
decreasing the classical processing load and complexity compared with currently favored schemes. They can be implemented by simple logical gates and do not need complex processors, and this would imply less heat dissipation into the system and could allow the classical control hardware to be closer to the qubits.

A widely used decoding algorithm for classical codes is based on iterative message passing on the Tanner graph and is called "belief propagation" (BP). The BP decoder is very appealing due to its simplicity, which could benefit hardware implementations, as well as its versatility, as it can in principle be applied to arbitrary quantum LDPC codes. Generally, BP does not work well when applied to Tanner graphs that contain small loops, a feature quantum codes necessarily have due to the commutativity constraint that introduces loops of length 4 (see Fig. 2). Furthermore, when applied to quantum codes, BP tends to fail to converge as there are many equivalent solutions up to the application of stabilizers. These problems were addressed in Refs. [106-111]. In particular, Duclos et al. [112] combined BP with a renormalization decoder and Panteleev and Kalachev [69] combined BP with ordered statistics decoding, which showed good performance on a variety of quantum LDPC code. BP decoders were analyzed in numerical simulations for tensor products of classical codes [113-115] and for 4D hyperbolic codes [51]. As BP is widely used for classical codes, one can draw from a wealth of literature. For example, there has been rapid progress on efficient hardware implementations of BP $[116,117]$.

For classical codes it has been observed that expansion properties of the Tanner graph can lead to simple greedy decoding algorithms [74]. Such greedy algorithms do not directly transfer to quantum codes. However, Leverrier et al. [97] found a suitable generalization, called the "small-set-flip decoder," that applies to tensor products of classical expander codes. Hastings [49] showed that the expansion properties of 4D hyperbolic codes can be used for decoding using a local greedy procedure as well.

Delfosse et al. [118] considered the union-find decoder, which was initially developed as an efficient decoder for the surface code, for decoding general quantum LDPC codes. Delfosse and Hastings [119] combined the unionfind decoder of the surface code [44] with a look-up decoder of a small code of fixed size, applying it to the tensor product of both codes. This raises the following question.

Open problem. Is there a systematic approach to generalize decoders of classical codes to work for quantum codes on the basis of their product?

Bounds on the optimal decoding performance for tensor products of random classical codes were given in Ref. [120].
A further potential advantage of LDPC codes over the surface code is single-shot decoding [121]. As stabilizer check measurements are subject to noise, they have to be repeated to build confidence [27]. Single-shot decoding refers to the property of some LDPC codes to exhibit robustness against such measurement errors, so it is not necessary to repeat the stabilizer check measurements. Numerical simulations of single-shot decoding were performed (under various assumptions and error models) for tensor product codes [122,123] and 4D hyperbolic codes [51].

\section{Hardware implementation}

A major concern often raised regarding the codes discussed in this paper is how they could be implemented in hardware. In the following section we discuss the main concerns and argue why we are optimistic about the potential of LDPC codes.

An important aspect of hardware implementation is that the maximum number of qubits involved in a stabilizer check should be low to keep the number of errors introduced low. Although this number is constant for LDPC codes by definition, it can still be too high for practical purposes, although it is possible to reduce the stabilizer check weight using graph-based arguments [65,124]. Higgott and Breuckmann [45] suggest an alternative construction by systematically breaking the stabilizer checks into smaller, so-called gauge checks, which do not commute, but from which the stabilizer check measurement can be inferred.

However, the most obvious drawback of LDPC codes comes with the question of how to lay out the physical qubits and their couplings in space. As a proxy, we discuss the layout of the Tanner graphs of codes. Almost none of the Tanner graphs of the quantum LDPC codes discussed here are planar, with the exception of a planar variation of hyperbolic surface codes [38]. More severely, several codes discussed here do not have "nice" embeddings in Euclidean space, as their Tanner graphs have a nontrivial expansion (although expanding graphs have been implemented in experiments [125]).

A related concern is that some qubit hardware implementations and their couplings are possible only in a planar layout. While planar embeddings of the discussed quantum LDPC codes are generally not possible, it is possible to break up graphs into planar pieces that are then connected along a one-dimensional line without intersections. This can be done using book embeddings [126], where the vertices of the graph are arranged along a line (spine) and each edge is assigned a half-plane with the line as its boundary (page), such that no two edges on the same page intersect. Clearly, the vertices do not have to be placed on the spine but can be pulled into the pages. The number of pages should ideally not grow, and it was shown in Ref. [127] that there are indeed families of expander graphs such that 
only three pages are sufficient for a book embedding. However, the minimum number of pages for the Tanner graphs of codes discussed here are not known to us.

The viability of implementing quantum codes requiring nonlocal couplings depends on the hardware. Currently, it is not settled which qubit architecture will succeed (see Ref. [128] for an overview). Hence, it is also not clear at this time how future quantum computing architectures will scale. Although some proposals suggest that a large number of physical qubits may be placed in a single fridge [129], it seems doubtful that arbitrary scaling inside a single fridge will be possible. Other proposals pursue a modular architecture of interconnected modules linked by a photonic interface [130-133]. A modular approach would free us from spacial constraints, making LDPC codes competitive candidates for implementing quantum fault tolerance. Other approaches to quantum computation, such as qubits coupled to a common cavity mode $[134,135]$, even allow direct, nonplanar interactions between qubits.

To measure the stabilizer checks it is necessary to find a scheduling, an ordering of the gates that couple the data qubits to an ancilla used for the measurement. This ordering should not spread errors so as be fault-tolerant and it should also be efficient to minimize the time of qubit idling. Finding such circuits is a nontrivial task and, as far as we are aware, hyperbolic surface codes are the only finite-rate codes that have a known measurement schedule $[45,136]$. Finding such schedules will be challenging for random constructions.

Open problem. Are there good measurement schedules for the LDPC codes discussed here?

\section{APPLICATIONS BEYOND QUANTUM ERROR CORRECTION}

We have seen that quantum LDPC codes draw from many areas of mathematics, physics, and computer science. One could hope that quantum LDPC codes could in turn find use beyond quantum error correction and quantum fault tolerance. Here we briefly highlight two examples where this is the case.

\section{A. Quantum complexity theory}

An important class in quantum complexity theory is QMA, an analog of the classical complexity class NP; see Ref. [137,138]. A prototypical QMA-complete problem is the $k$-local Hamiltonian problem); see Ref. [137]. It asks whether the ground-state energy of a $k$-local Hamiltonian is either below $a$ or above $b$, where $b-a>1 /$ poly $n$, and can be seen as the quantum analog of 3-SAT. One of the main achievements of classical complexity theory, the PCP theorem, also a admits a conjectural quantum version. The quantum PCP (QPCP) conjecture states that the local Hamiltonian problem is equally hard when stated with a constant accuracy $b-a>$ const instead of an inverse-polynomial accuracy; see Refs. [139,140].

Hastings introduced the no low-energy trivial state (NLTS) conjecture, a weakening of the QPCP conjecture $[141,142]$. It states that there is family of local Hamiltonians acting on an increasing number of qubits such that the energy of any trivial state is below a universal constant. The NLTS conjecture could be solved by construction of quantum LDPC codes with a linear distance for which there exist local Hamiltonians for which the energy of a quantum state is proportional to its distance from the ground space of the Hamiltonian (quantum locally testable codes). Toward solving the NLTS conjecture, Anshu and Nirkhe [143] showed that quantum LDPC codes with a linear rate and polynomial distance have no trivial states of energy less than $o(n)$. See Ref. [144] for a zoo of the various complexity classes and their relation to quantum LDPC codes and Ref. [145] for a comprehensive review of the QPCP and NLTS conjectures.

\section{B. Geometry}

In Sec. III we saw that quantum LDPC codes can be constructed using tools from geometry. More precisely, quantum codes can be defined from tessellations of manifolds such that the code properties are determined by the geometric properties. Recently, Freedman and Hastings [146] showed that the inverse is also possible. Given a quantum LDPC code, they constructed manifolds of dimension $D=11$ such that geometric properties of the manifold are determined by the properties of the code. Their work suggests that questions of systolic geometry can be answered using quantum LDPC code constructions.

\section{CONCLUSION AND OUTLOOK}

In this Perspective, we gave an overview of the emerging field of quantum LDPC codes, providing promising new approaches to quantum error correction. Quantum LDPC codes use a plethora of techniques from mathematics, physics, and computer science. In particular, we showed how ideas from geometry and homological algebra shape the theory of quantum LDPC codes. The results discussed here use hyperbolic geometry, expander codes, and algebraic topology, to name a few methods. The fast pace of new distance records in the last year suggests that one of the main goals of the field, the quest for quantum LDPC codes with constant encoding rate $k / n>$ const $>0$ and linear distance $d \propto n$, may soon be in reach and that the next few years may offer many exciting new developments.

Moreover, we discussed challenges and opportunities. In particular, the viability of quantum LDPC codes depends on future developments in hardware, and many problems in the implementation of scalable fault-tolerant quantum computation remain to be solved. Low-latency classical 
control and fast decoding algorithms as well as interconnectivity and wiring are challenging problems for the architecture of scalable quantum devices. Quantum LDPC codes could play a decisive role in their realization. Although the development of the surface code is ahead in many respects, quantum LDPC codes may well turn out to be better suited for the implementation of quantum computers in the mid term to long term.

On a theoretical level, quantum LDPC codes may yield exciting applications in geometry, quantum complexity theory, and potentially beyond, indicating that the flow of ideas can be reversed.

\section{ACKNOWLEDGMENTS}

The authors thank Matt Hastings, Gleb Kalachev, Anirudh Krishna, Alex Lubotzky, Pavel Panteleev, Joschua Ramette, and Christophe Vuillot for helpful discussions. They especially thank Barbara Terhal for valuable feedback on the manuscript. N.P.B. acknowledges support through the EPSRC Prosperity Partnership in Quantum Software for Simulation and Modelling (Grant No. EP/S005021/1).

\section{APPENDIX A: CONSTRUCTIONS FOR CHAIN COMPLEXES}

For the convenience of the reader, we describe various homological constructions, such as the different product codes from Sec. IV A, in greater detail.

\section{Chain complexes}

A chain complex $C=\left(C, \partial^{C}\right)$ of vector spaces over $\mathbb{F}_{2}$ of length $n+1$ is a collection of vector spaces $C_{i}$ and linear maps $\partial_{i}^{C}$, called "boundary operators,"

$$
C=\left(C_{n} \stackrel{\partial_{n}^{C}}{\longrightarrow} \cdots \stackrel{\partial_{2}^{C}}{\longrightarrow} C_{1} \stackrel{\partial_{1}^{C}}{\longrightarrow} C_{0}\right)
$$

fulfilling $\partial_{i+1}^{C} \partial_{i}^{C}=0$. Often, the indices of boundary operators are suppressed in the notation. For example, one simply writes $\partial^{2}=0$.

With a chain complex $C$ one can associate the vector spaces

$$
\begin{aligned}
& Z_{i}(C)=\operatorname{ker} \partial_{i} \subset C_{i} \\
& B_{i}(C)=\operatorname{im} \partial_{i+1} \subset C_{i} \\
& H_{i}(C)=Z_{i}(C) / B_{i}(C),
\end{aligned}
$$

of $i$-cycles, $i$-boundaries, and the $i$ th homology, respectively, whereas elements in $C_{i}$ are called " $i$-chains."

We assume that the spaces $C_{i}$ are equipped with bases of so-called $i$-cells. This defines scalar products on each $C_{i}$. We denote the linear dual of $C_{i}$ by $C^{i}$. Elements in $C^{i}$ are called "cochains." The canonical basis allows us to identify $C_{i}=C^{i}$, and one defines the vector spaces

$$
\begin{aligned}
Z^{i}(C) & =\operatorname{ker} \partial_{i+1}^{\mathrm{tr}} \subset C^{i}, \\
B^{i}(C) & =\operatorname{im} \partial_{i}^{\mathrm{tr}} \subset C^{i}, \\
H^{i}(C) & =Z^{i}(C) / B^{i}(C),
\end{aligned}
$$

of $i$-cocycles, $i$-coboundaries, and the $i$ th cohomology of the complex $C$. The scalar product on $C_{i}$ and $C^{i}$ induces a well-defined and nondegenerate pairing of $H_{i}(C)$ and $H^{i}(C)$ since $B^{i}(C)=Z_{i}(C)^{\perp}$. This implies that $\operatorname{dim} H_{i}(C)=\operatorname{dim} H^{i}(C)$.

For each $i$, a quantum code can be extracted from the cell complex $C$ with parity-check matrices $H_{X}$ and $H_{Z}$ corresponding to the operators $\partial_{i}$ and $\partial_{i+1}^{\mathrm{tr}}$. The $X$-checks, physical qubits, and $Z$-checks then correspond to the $i+1$ cells, $i$-cells, and $i-1$-cells of $C$. The nontrivial logical $X$-operators and $Z$-operators correspond to the elements in $H^{i}(C)$ and $H_{i}(C)$, respectively. See also Sec. II B 1 for the relation of chain complexes and (quantum) codes.

\section{Total complex of double complexes}

An interesting way of constructing chain complexes is by the total complex construction of a double complex. A double complex $E=\left(E_{\bullet, \bullet}, \partial^{v}, \partial^{h}\right)$ is an array of vector spaces $E_{p, q}$ equipped with vertical and horizontal maps,

$$
\begin{aligned}
& \partial_{p, q}^{v}: E_{p, q} \rightarrow E_{p, q-1} \\
& \text { and } \\
& \partial_{p, q}^{h}: E_{p, q} \rightarrow E_{p-1, q},
\end{aligned}
$$

such that $\partial^{v}$ and $\partial^{h}$ are commuting boundary operators

$$
\left(\partial^{v}\right)^{2}=\left(\partial^{h}\right)^{2}=0 \quad \text { and } \quad \partial^{v} \partial^{h}=\partial^{h} \partial^{v} .
$$

It is convenient to visualize the double complex laid out on a two-dimensional grid where each square is required

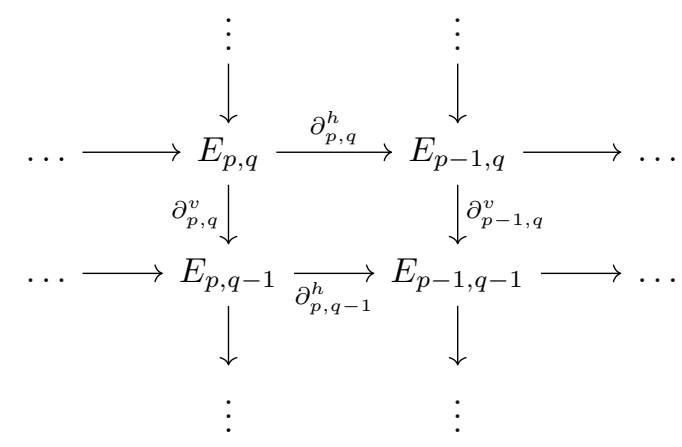

FIG. 9. A part of a double complex. 
to commute and composing two maps in the same direction yields zero; see Fig. 9. With each double complex $E$, one can associate a chain complex $\operatorname{Tot}(E)$, called the "total complex," where the $n$th degree is given by the direct sum over the $n$th diagonal in $E$, so

$$
\operatorname{Tot}(E)_{n}=\bigoplus_{p+q=n} E_{p, q}
$$

and the boundary operators of $\operatorname{Tot}(E)$ are the sum of all boundary operators passing from one diagonal to the next. The requirement that the boundary operators of $\operatorname{Tot}(E)$ square to zero directly follows from Eq. (A1).

These concepts immediately generalize to higher dimensions. See Fig. 11 for an example of a triple complex.

\section{Tensor product of chain complexes and hypergraph products}

Let $C$ and $D$ be complexes of length $n$ and $m$, respectively. The tensor product $C \otimes D$ is a chain complex of length $n+m-1$ and can be seen as a generalization of the tensor product of vector spaces to complexes.

There is an elegant and quick definition of tensor product $C \otimes D$ in terms of double complexes. Namely, the tensor product double complex $C \otimes D$ is defined by

$$
(C \otimes D)_{p, q}=C_{p} \otimes C_{q},
$$

with boundary operators $\partial^{v}=\partial^{C} \otimes \operatorname{id}_{D}$ and $\partial^{h}=\operatorname{id}_{C} \otimes \partial^{D}$. After a basis has been chosen, the tensor product of two maps is given by the Kronecker product of the corresponding matrices. Then $C \otimes D=\operatorname{Tot}(C \otimes D)$ is the total complex of this double complex.

For example, if $C$ and $D$ are chain complexes of length 2 , then the tensor product $C \otimes D$ of $C$ and $D$ is a chain complex of length 3 with boundary operators

$$
\left(\begin{array}{l}
\partial_{1}^{C} \otimes \mathrm{id}_{D_{1}} \\
\operatorname{id}_{C_{1}} \otimes \partial_{1}^{D}
\end{array}\right) \quad \text { and } \quad\left(\operatorname{id}_{C_{0}} \otimes \partial_{1}^{D}, \quad \partial_{1}^{C} \otimes \operatorname{id}_{D_{0}}\right)
$$

respectively. The relation between this direct definition and the definition via total complexes is visualized in Fig. 10.

The tensor product of chain complexes can be used to define the hypergraph product of two classical codes. Assume that the codes have $n_{i}$ bits and $r_{i}$ checks for $i=$ 1,2 and parity-check matrices $H$ and $H^{\prime}$. With the codes one associates two chain complexes $C$ and $D$ of length 2 with boundary operators $\partial_{C}^{1}$ and $\partial_{D}^{1}$ represented by the matrices $H$ and $\left(H^{\prime}\right)^{\text {tr }}$. Then the hypergraph product arises as the tensor product $C \otimes D$. In particular, the parity-check matrices $H_{Z}^{\mathrm{tr}}$ and $H_{X}$ of the hypergraph product code are

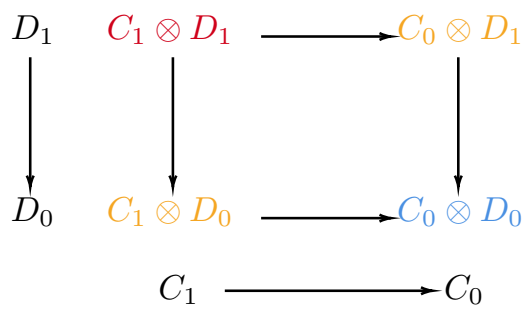

FIG. 10. A double complex arising as the tensor product of two complexes of length 2 . The different colors symbolize the different degrees in the total complex, which is a chain complex of length 3 . The diagram should be compared with the diagram in Fig. 6.

given by

$$
\left(\begin{array}{c}
H \otimes I_{r_{2}} \\
I_{n_{1}} \otimes\left(H^{\prime}\right)^{\mathrm{tr}}
\end{array}\right) \quad \text { and } \quad\left(I_{r_{1}} \otimes\left(H^{\prime}\right)^{\mathrm{tr}}, \quad H \otimes I_{n_{2}}\right) .
$$

The homology of a tensor product is the subject of the Künneth formula

$$
H_{n}(C \otimes D)=\bigoplus_{p+q=n} H_{p}(C) \otimes H_{q}(D)
$$

This allows us to easily compute the number of logical qubits of a tensor product or hypergraph product quantum code.

Moreover, one can also take iterated tensor products of chain complexes, which correspond to higher-dimensional complexes. See Fig. 11 for an example of a tensor product of three complexes.

\section{Fiber bundle codes}

In topology, a fiber bundle is generalization of a product of two spaces, which allows nontrivial twists. It consists of a projection map $\pi: E \rightarrow B$ from its total space to its base, such that the fibers $F=\pi^{-1}(x)$ are isomorphic and $E$ is a product of the base and the fiber locally. A prototypical example of a fiber bundle is the Klein bottle, which admits a map to a circle whose fiber is also a circle. The Klein

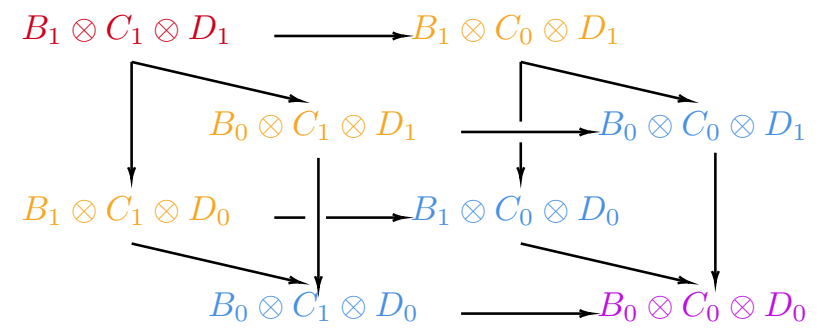

FIG. 11. A triple complex arising as the tensor product of three complexes of length 3 . The different colors symbolize the different degrees in the total complex, which is a chain complex of length 4 . 
bottle is a twisted version of a product of two circles, the torus.

Fiber bundle codes mimic this topological concept and were introduced by Hastings et al. [68] to build quantum LDPC codes breaking the $\sqrt{n}$ polylog $n$ distance barrier. The idea behind fiber bundle codes is to introduce a twist in the boundary operators of tensor product codes so as to increase the distance of the resulting code.

Let $B$ and $F$ be two complexes of length 2 equipped with bases; we will refer to these as base and fiber complex, respectively. Furthermore, let $\varphi$ be a function (called "twist") that associates with any pair of incident basis vectors of $B_{0}$ and $B_{1}$ an automorphism of the fiber.

Then the fiber bundle code $B \otimes_{\varphi} F$ is a chain complex with the same underlying vector spaces as $B \otimes F$ but with twisted boundary operators

$$
\left(\begin{array}{c}
\partial_{\varphi} \\
\operatorname{id}_{B_{1}} \otimes \partial_{1}^{F}
\end{array}\right) \quad \text { and } \quad\left(\begin{array}{ll}
\partial_{\varphi} & \partial_{1}^{B} \otimes \mathrm{id}_{F_{0}}
\end{array}\right),
$$

where

$$
\partial_{\varphi}\left(b^{1} \otimes f\right)=\sum_{b^{0} \in \partial^{B} b^{1}} b^{0} \otimes \varphi\left(b^{1}, b^{0}\right)(f),
$$

for basis vectors $b_{i} \in B_{i}$ and $f \in F_{0}$ or $f \in F_{1}$. In particular, if $\varphi=1$ then the fiber bundle $B \otimes_{\varphi} F=B \otimes F$ specializes to the tensor product. The fiber bundle code $B \otimes_{\varphi} F$ can be interpreted as the total complex of the fiber bundle double complex $B \otimes_{\varphi} F$ with the obvious boundary operators.

Requiring that $\partial_{1}^{B}$ is surjective and some additional technical conditions [68], one can show that

$$
H^{1}\left(B \otimes_{\varphi} F\right)=H^{1}(B) .
$$

Hence the number of logical qubits in $B \otimes_{\varphi} F$ coincides with the number of encoded bits in the code associated the complex $B$. In Ref. [68] the construction was applied to a random code $B$ as the base, a repetition code with cyclic symmetry $F$ as the fiber, and random twist $\varphi$.

\section{Lifted product codes}

Lifted product codes, introduced by Panteleev and Kalachev [57,69] are based on the observation that the tensor product of vector spaces or the Kronecker product of matrices extends to modules over algebras. This more general definition can be used to construct quantum codes.

Let $R \subset \mathbb{F}_{2}^{\ell \times \ell}$ be a commutative subalgebra of the ring of $\ell \times \ell$ matrices over $\mathbb{F}_{2}$. Let $A \in R^{n \times m}$ and $B \in R^{k \times l}$ be matrices with entries in the algebra $R$. Equivalently, $A$ and $B$ can be interpreted as matrices $\tilde{A} \in \mathbb{F}_{2}^{\ell n \times \ell m}$ and $\tilde{B} \in \mathbb{F}_{2}^{\ell k \times \ell l}$ whose blocks of size $\ell \times \ell$ are elements in the algebra $R$.
Then the lifted product quantum code is defined in terms of the check matrices $H_{X}$ and $H_{Z}$ given by

$$
\left(I_{m}^{R} \otimes_{R} B \quad A \otimes_{R} I_{l}^{R}\right) \quad \text { and } \quad\left(A \otimes_{R} I_{k}^{R} \quad I_{n}^{R} \otimes_{R} B\right),
$$

where $\otimes_{R}$ denotes the Kronecker product of matrices over $R, I_{q}^{R}$ denotes the $q \times q$ identity matrix over $R$, and the resulting matrices are interpreted as matrices over $\mathbb{F}_{2}$.

Equivalently, the parity-check matrices and $H_{X}$ and $H_{Z}$ can be obtained by first considering the matrices

$$
\left(I_{\ell m} \otimes \tilde{B} \quad \tilde{A} \otimes I_{\ell l}\right) \quad \text { and } \quad\left(\tilde{A} \otimes I_{\ell k} \quad I_{\ell n} \otimes \tilde{B}\right)
$$

using the usual Kronecker product. The resulting matrices can be subdivided into submatrices of size $\ell^{2} \times \ell^{2}$. Then one collapses each of these these submatrices to matrices of size $\ell \times \ell$ by adding all of their $\ell \times \ell$ submatrices of size $\ell \times \ell$ together.

The number of $X$-checks, $Z$-checks, and logical qubits in the lifted product is smaller by a factor of $\ell$ than in the corresponding tensor (hypergraph) product.

The lifted product can also be written as a tensor product of chain complexes. Here one has to interpret the two classical codes as chain complexes of length 2 over the algebra $R$ and use the tensor product over $R$. This is closely related to the definition of balanced product codes; see Sec. VIII A f.

In Refs. [57,69] the lifted product construction is applied mostly in the case where $R$ is the algebra of circulant matrices; that is, the algebra generated by the cyclic shift matrix $x$ of the $\ell$-cycle. Matrices with entries in $R$ can be constructed, for example, from the incidence matrix of a graph with an $\ell$-fold cyclic covering (see Ref. [147]) and associated expander codes. Indeed, quantum LDPC codes with an almost linear distance were obtained in Ref. [57] by taking the lifted product of a Sipser-Spielman code on a random cyclic covering of a random expander graph with the matrix $1+x$ of the repetition code.

\section{Balanced product codes}

The balanced product is a topological construction that associates with two spaces $X, Y$ with right and left actions of a groups $G$, respectively, a space $X \times_{G} Y$. The space $X \times{ }_{G} Y$ is defined as the quotient $(X \times Y) / G$ of the Cartesian product, where $G$ acts on $X \times Y$ via $g \cdot(x, y)=$ $\left(x g^{-1}, g y\right)$. The balanced product is often used to construct fiber bundles from principal bundles in physics and topology; see Ref. [37]. Namely, the natural projection $\pi: X \times_{G} Y \rightarrow X / G$ is a fiber bundle with base $X / G$ and fiber $Y$, under some technical assumption.

Balanced product codes mimic this concept and were introduced by Breuckmann and Eberhardt [70] to build quantum LDPC codes.

If $G$ is a group acting on a vector space $V$ and a vector space $W$ from the left and right, respectively, one can form 
the tensor product over $G$ via

$$
V \otimes_{G} W=V \otimes W /\langle v \cdot g \otimes w-v \otimes g \cdot w\rangle .
$$

Similarly to the tensor product for vector spaces, this definition extends to chain complexes. Let $C$ and $D$ be chain complexes with a right and left action of $G$, respectively. In other words, $G$ acts on the individual spaces $C_{i}$ and $D_{i}$ and commutes with all boundary operators. Then one can form the complex $C \otimes_{G} D$, which is the total complex of the double complex $C \nabla_{G} D$.

Under the assumption that $G$ is a finite group of odd order, there is a Künneth formula

$$
H_{n}\left(C \otimes_{G} D\right)=\bigoplus_{p+q=n} H_{p}(C) \otimes_{G} H_{q}(D) .
$$

In the case that $G$ is a commutative group with a free action on each vector space $C_{i}$ and $D_{i}$, the balanced product specializes to a lifted product.

The balanced product construction was applied by Breuckmann and Eberhardt to construct quantum LDPC codes from highly symmetrical Sipser-Spielman codes and a repetition code with cyclic symmetry. To construct Sipser-Spielman codes, Breuckmann and Eberhardt used Cayley graphs of $\operatorname{PGL}(2, q)$, whose automorphism is exactly this group.

\section{Relation of fiber bundle and balanced product codes}

As mentioned above, fiber bundles and balanced products are closely related concepts in topology. Similarly, balanced product, lifted product, and fiber bundle codes are closely related; see Fig. 8 . The code families breaking the $\sqrt{n}$ polylog $n$ distance barrier described in Sec. IV A can be interpreted in all three setups.

We illustrate the relationship with a topological example. Denote by $X=S^{1}$ the circle and let $G=\mathbb{Z}_{2}$ act on $X$ via a rotation by $\pi$. The quotient space $X / G=S^{1}$ is also a circle and $\pi: X \rightarrow X / H$ is a twofold covering. Let $G$ act on another circle $Y=S^{1}$ by reflection along the $x$ axis. Then the associated balanced product $S^{1} \times_{\mathbb{Z}_{2}} S^{1}$ is a Klein bottle, which is a fiber bundle over the circle

$$
\pi_{S^{1}}: S^{1} \times_{\mathbb{Z}_{2}} S^{1} \rightarrow S^{1}
$$

with fiber $S^{1}$. By choosing $G$-equivariant tessellations of $X$ and $Y$, one obtains quantum codes that can be interpreted as balanced product, lifted product, and fiber bundle codes. Similarly, it is often possible to relate similar such code constructions to each other.

[1] F. Arute, K. Arya, R. Babbush, D. Bacon, J. C. Bardin, R. Barends, R. Biswas, S. Boixo, F. G. Brandao, D. A.
Buell, et al., Quantum supremacy using a programmable superconducting processor, Nature 574, 505 (2019).

[2] H.-S. Zhong, H. Wang, Y.-H. Deng, M.-C. Chen, L.-C. Peng, Y.-H. Luo, J. Qin, D. Wu, X. Ding, Y. Hu, et al., Quantum computational advantage using photons, Science 370, 1460 (2020).

[3] P. Jurcevic, A. Javadi-Abhari, L. S. Bishop, I. Lauer, D. Borgorin, M. Brink, L. Capelluto, O. Gunluk, T. Itoko, N. Kanazawa, et al., Demonstration of quantum volume 64 on a superconducting quantum computing system, Quantum Sci. Technol. 6, 025020 (2021).

[4] A. Schönhage, in International Colloquium on Automata, Languages, and Programming (Springer, Graz, Austria, 1979), p. 520.

[5] R. Landauer, The physical nature of information, Phys. Lett. A 217, 188 (1996).

[6] S. Aaronson, Quantum Computing Since Democritus (Cambridge University Press, Cambridge, UK, 2013).

[7] P. W. Shor, Scheme for reducing decoherence in quantum computer memory, Phys. Rev. A 52, R2493 (1995).

[8] A. Y. Kitaev, Quantum computations: Algorithms and error correction, Usp. Mat. Nauki 52, 53 (1997).

[9] A. Y. Kitaev, Fault-tolerant quantum computation by anyons, Ann. Phys. (NY) 303, 2 (2003).

[10] M. H. Freedman and D. A. Meyer, Projective plane and planar quantum codes, Found. Comput. Math. 1, 325 (2001).

[11] S. B. Bravyi and A. Y. Kitaev, Quantum codes on a lattice with boundary, ArXiv:quant-ph/9811052 (1998).

[12] Z. Chen, K. J. Satzinger, J. Atalaya, A. N. Korotkov, A. Dunsworth, D. Sank, C. Quintana, M. McEwen, R. Barends, P. V. Klimov, et al., Exponential suppression of bit or phase flip errors with repetitive error correction, ArXiv:2102.06132 (2021).

[13] A. R. Calderbank and P. W. Shor, Good quantum errorcorrecting codes exist, Phys. Rev. A 54, 1098 (1996).

[14] A. Ashikhmin, S. Litsyn, and M. A. Tsfasman, Asymptotically good quantum codes, Phys. Rev. A 63, 032311 (2001).

[15] R. Gallager, Low-density parity-check codes, IRE Trans. Inf. Theory 8, 21 (1962).

[16] D. J. MacKay and R. M. Neal, Near Shannon limit performance of low density parity check codes, Electron. Lett. 32, 1645 (1996).

[17] T. B. Iliev, G. V. Hristov, P. Z. Zahariev, and M. P. Iliev, in Novel Algorithms and Techniques In Telecommunications, Automation and Industrial Electronics (Springer, Heidelberg, Germany, 2008), p. 532.

[18] D. Gottesman, Fault-tolerant quantum computation with constant overhead, Quantum Inf. Comput. 14, 1338 (2014).

[19] E. Knill, R. Laflamme, and W. H. Zurek, Resilient quantum computation: Error models and thresholds, Proc. R. Soc. London, A: Math. Phys. Eng. Sci. 454, 365 (1998).

[20] D. Aharonov and M. Ben-Or, Fault-tolerant quantum computation with constant error rate, SIAM J. Comput. 38, 1207 (2008).

[21] M. M. Wilde, Quantum Information Theory (Cambridge University Press, Cambridge, UK, 2013). 
[22] L. Gyongyosi, S. Imre, and H. V. Nguyen, A survey on quantum channel capacities, IEEE Commun. Surv. Tutor. 20, 1149 (2018).

[23] N. Delfosse and G. Zémor, Upper bounds on the rate of low density stabilizer codes for the quantum erasure channel, ArXiv:1205.7036 (2012).

[24] T. Richardson and R. Urbanke, Modern Coding Theory (Cambridge University Press, Cambridge, UK, 2008).

[25] D. J. MacKay, G. Mitchison, and P. L. McFadden, Sparsegraph codes for quantum error correction, IEEE Trans. Inf. Theory 50, 2315 (2004).

[26] D. A. Lidar and T. A. Brun, Quantum Error Correction (Cambridge University Press, Cambridge, UK, 2013).

[27] E. Dennis, A. Kitaev, A. Landahl, and J. Preskill, Topological quantum memory, J. Math. Phys. 43, 4452 (2002).

[28] A. M. Kubica, Ph.D. thesis, California Institute of Technology (2018).

[29] B. M. Terhal, Quantum error correction for quantum memories, Rev. Mod. Phys. 87, 307 (2015).

[30] J. Preskill, Lecture notes for physics 229: Quantum information and computation, Cal. Inst. Technol. 16, 10 (1998).

[31] D. Gottesman, Ph.D. thesis, Caltech, ArXiv:quant-ph/ 9705052 (1997).

[32] A. R. Calderbank, E. M. Rains, P. Shor, and N. J. Sloane, Quantum error correction via codes over GF(4), IEEE Trans. Inf. Theory 44, 1369 (1998).

[33] S. Bravyi, B. M. Terhal, and B. Leemhuis, Majorana fermion codes, New J. Phys. 12, 083039 (2010).

[34] H. Bombín and M. A. Martin-Delgado, Homological error correction: Classical and quantum codes, J. Math. Phys. 48, 052105 (2007).

[35] A. Hatcher, Algebraic Topology (Cambridge University Press, Cambridge, UK, 2002).

[36] N. P. Breuckmann, Ph.D. thesis, RWTH Aachen University (2017).

[37] M. Nakahara, Geometry, Topology and Physics (CRC press, Boca Raton, US, 2003).

[38] N. P. Breuckmann and B. M. Terhal, Constructions and noise threshold of hyperbolic surface codes, IEEE Trans. Inf. Theory 62, 3731 (2016).

[39] N. P. Breuckmann, C. Vuillot, E. Campbell, A. Krishna, and B. M. Terhal, Hyperbolic and semi-hyperbolic surface codes for quantum storage, Quantum Sci. Technol. 2, 035007 (2017).

[40] I. H. Kim, Bachelor's Thesis, Massachusetts Institute of Technology (2007).

[41] G. Zémor, in International Conference on Coding and Cryptology (Springer, Heidelberg, Germany, 2009), p. 259.

[42] N. Delfosse, in 2013 IEEE International Symposium on Information Theory (IEEE, Istanbul, Turkey, 2013), p. 917.

[43] A. A. Kovalev and L. P. Pryadko, Fault tolerance of quantum low-density parity check codes with sublinear distance scaling, Phys. Rev. A 87, 020304 (2013).

[44] N. Delfosse and N. H. Nickerson, Almost-linear time decoding algorithm for topological codes, ArXiv:1709. 06218 (2017).

[45] O. Higgott and N. P. Breuckmann, Subsystem codes with high thresholds by gauge fixing and reduced qubit overhead, ArXiv:2010.09626 (2020).
[46] W. S. Soares Jr and E. B. Da Silva, Hyperbolic quantum color codes, ArXiv:1804.06382 (2018).

[47] C. Vuillot and N. P. Breuckmann, Quantum pin codes, ArXiv:1906.11394 (2019).

[48] L. Guth and A. Lubotzky, Quantum error correcting codes and 4-dimensional arithmetic hyperbolic manifolds, J. Math. Phys. 55, 082202 (2014).

[49] M. B. Hastings, Decoding in hyperbolic spaces: LDPC codes with linear rate and efficient error correction, Quantum Inf. Comput. 14, 1187 (2014).

[50] V. Londe and A. Leverrier, Golden codes: Quantum LDPC codes built from regular tessellations of hyperbolic 4manifolds, Quantum Inf. Comput 19, 0361 (2019).

[51] N. P. Breuckmann and V. Londe, Single-shot decoding of linear rate LDPC quantum codes with high performance, ArXiv:2001.03568 (2020).

[52] M. H. Freedman, D. A. Meyer, and F. Luo, Z2-systolic freedom and quantum codes, Mathematics of Quantum Computation, edited by Ranee K. Brylinski and Goong Chen (Chapman \& Hall/CRC, Boca Raton, US, 2002), Vol. 287, p. 287.

[53] There is an unfortunate typographical error in Ref. [52], claiming distance scaling as $\Omega(\sqrt[2]{\log n} \sqrt{n})$.

[54] E. Fetaya, Master's thesis, The Hebrew University of Jerusalem, ArXiv:1108.2886 (2011).

[55] J. Haah, Algebraic methods for quantum codes on lattices, Rev. Colomb. Mat. 50, 299 (2016).

[56] J. Haah, Local stabilizer codes in three dimensions without string logical operators, Phys. Rev. A 83, 042330 (2011).

[57] P. Panteleev and G. Kalachev, Quantum LDPC codes with almost linear minimum distance, ArXiv:2012.04068 (2020).

[58] B. J. Brown, D. Loss, J. K. Pachos, C. N. Self, and J. R. Wootton, Quantum memories at finite temperature, Rev. Mod. Phys. 88, 045005 (2016).

[59] E. Fetaya, Bounding the distance of quantum surface codes, J. Math. Phys. 53, 062202 (2012).

[60] S. Bravyi, D. Poulin, and B. Terhal, Tradeoffs for Reliable Quantum Information Storage in 2D Systems, Phys. Rev. Lett. 104, 050503 (2010).

[61] T. Devakul and D. J. Williamson, Fractalizing quantum codes, Quantum 5, 438 (2021).

[62] M. G. Katz, Systolic Geometry and Topology (American Mathematical Soc., Providence, US, 2007), Vol. 137.

[63] J.-P. Tillich and G. Zémor, Quantum LDPC codes with positive rate and minimum distance proportional to the square root of the blocklength, IEEE Trans. Inf. Theory 60, 1193 (2013).

[64] S. Bravyi and M. B. Hastings, in Proceedings of the FortySixth Annual ACM Symposium on Theory of Computing (Association for Computing Machinery New York, US, 2014), p. 273.

[65] M. B. Hastings, Weight reduction for quantum codes, ArXiv:1611.03790 (2016).

[66] S. Evra, T. Kaufman, and G. Zémor, Decodable quantum LDPC codes beyond the $\sqrt{n}$ distance barrier using high dimensional expanders, ArXiv:2004.07935 (2020).

[67] T. Kaufman and R. J. Tessler, New cosystolic expanders from tensors imply explicit quantum LDPC codes with $\omega\left(\sqrt{n} \log ^{k} n\right)$ distance, ArXiv:2008.09495 (2020). 
[68] M. B. Hastings, J. Haah, and R. O'Donnell, Fiber bundle codes: Breaking the $n^{1} / 2$ polylog $(n)$ barrier for quantum LDPC codes, ArXiv:2009.03921 (2020).

[69] P. Panteleev and G. Kalachev, Degenerate quantum LDPC codes with good finite length performance, ArXiv:1904.02703 (2019).

[70] N. P. Breuckmann and J. N. Eberhardt, Balanced product quantum codes (2020), ArXiv:2012.09271.

[71] C. A. Weibel, An Introduction to Homological Algebra, Cambridge Studies in Advanced Mathematics (Cambridge University Press, Cambridge, 1994).

[72] A. Leverrier, J. Tillich, and G. Zémor, in 2015 IEEE 56th Annual Symposium on Foundations of Computer Science (IEEE Computer Society Conference Publishing Services, Washington DC, US, 2015), p. 810, ISSN: 0272-5428.

[73] O. Fawzi, A. Grospellier, and A. Leverrier, in 2018 IEEE 59th Annual Symposium on Foundations of Computer Science (FOCS) (IEEE Computer Society Conference Publishing Services, Washington DC, US, 2018), p. 743, ISSN: 2575-8454.

[74] M. Sipser and D. A. Spielman, Expander codes, IEEE Trans. Inf. Theory 42, 1710 (1996), conference Name: IEEE Transactions on Information Theory.

[75] A. A. Kovalev and L. P. Pryadko, in 2012 IEEE International Symposium on Information Theory Proceedings (IEEE Computer Society Conference Publishing Services, Washington DC, US, 2012), p. 348, ISSN: 2157-8117.

[76] W. Zeng and L. P. Pryadko, Higher-Dimensional Quantum Hypergraph-Product Codes with Finite Rates, Phys. Rev. Lett. 122, 230501 (2019), publisher: American Physical Society.

[77] B. Audoux and A. Couvreur, On tensor products of CSS codes, Ann. Inst. Henri Poincaré (D) Combinat. Phys. Interact. 6, 239 (2019), publisher: European Mathematical Society.

[78] A. Lubotzky, High dimensional expanders, ArXiv:1712. 02526 (2017).

[79] A. Lubotzky, R. Phillips, and P. Sarnak, Ramanujan graphs, Combinatorica 8, 261 (1988).

[80] A. Leverrier, S. Apers, and C. Vuillot, Quantum XYZ product codes, ArXiv:2011.09746 (2020).

[81] D. Maurice, Ph.D. thesis, Université Pierre et Marie CurieParis VI (2014).

[82] C. Chamon, Quantum Glassiness in Strongly Correlated Clean Systems: An Example of Topological Overprotection, Phys. Rev. Lett. 94, 040402 (2005).

[83] S. Bravyi, B. Leemhuis, and B. M. Terhal, Topological order in an exactly solvable 3D spin model, Ann. Phys. (NY) 326, 839 (2011).

[84] J. P. Bonilla-Ataides, D. K. Tuckett, S. D. Bartlett, S. T. Flammia, and B. J. Brown, The XZZX surface code, ArXiv:2009.07851 (2020).

[85] M. B. Hastings, Quantum codes from high-dimensional manifolds, ArXiv:1608.05089 (2016).

[86] S. Bravyi, Subsystem codes with spatially local generators, Phys. Rev. A 83, 012320 (2011).

[87] T. J. Yoder, Optimal quantum subsystem codes in two dimensions, Phys. Rev. A 99, 052333 (2019).

[88] M. Li and T. J. Yoder, in 2020 IEEE International Conference on Quantum Computing and Engineering (QCE) (IEEE, 2020), p. 109.
[89] D. Bacon, S. T. Flammia, A. W. Harrow, and J. Shi, Sparse quantum codes from quantum circuits, IEEE Trans. Inf. Theory 63, 2464 (2017).

[90] A. R. Calderbank and P. W. Shor, Good quantum errorcorrecting codes exist, Phys. Rev. A 54, 1098 (1996).

[91] T. C. Bohdanowicz, E. Crosson, C. Nirkhe, and H. Yuen, in Proceedings of the 51st Annual ACM SIGACT Symposium on Theory of Computing (Association for Computing Machinery, New York, US, 2019), p. 481.

[92] W. Brown and O. Fawzi, in 2013 IEEE International Symposium on Information Theory (IEEE, Istanbul, Turkey, 2013), p. 346.

[93] N. P. Breuckmann and B. M. Terhal, Space-time circuitto-hamiltonian construction and its applications, J. Phys. A: Math. Theor. 47, 195304 (2014).

[94] R. Movassagh and Y. Ouyang, Constructing quantum codes from any classical code and their embedding in ground space of local hamiltonians, ArXiv:2012.01453 (2020).

[95] E. Knill, Quantum computing with realistically noisy devices, Nature 434, 39 (2005).

[96] O. Fawzi, A. Grospellier, and A. Leverrier, in 2018 IEEE 59th Annual Symposium on Foundations of Computer Science (FOCS) (IEEE, Paris, France, 2018), p. 743.

[97] A. Leverrier, J.-P. Tillich, and G. Zémor, in 2015 IEEE 56th Annual Symposium on Foundations of Computer Science (IEEE, Berkeley, US, 2015), p. 810.

[98] E. T. Campbell, B. M. Terhal, and C. Vuillot, Roads towards fault-tolerant universal quantum computation, Nature 549, 172 (2017).

[99] S. Bravyi and R. König, Classification of Topologically Protected Gates for Local Stabilizer Codes, Phys. Rev. Lett. 110, 170503 (2013).

[100] F. Pastawski and B. Yoshida, Fault-tolerant logical gates in quantum error-correcting codes, Phys. Rev. A 91, 012305 (2015).

[101] A. Krishna and D. Poulin, Fault-tolerant gates on hypergraph product codes, ArXiv:1909.07424 (2019).

[102] S. Burton and D. Browne, Limitations on transversal gates for hypergraph product codes, ArXiv:2012.05842 (2020).

[103] T. Jochym-O'Connor, Fault-tolerant gates via homological product codes, Quantum 3, 120 (2019).

[104] P. Iyer and D. Poulin, Hardness of decoding quantum stabilizer codes, IEEE Trans. Inf. Theory 61, 5209 (2015).

[105] P. Das, C. A. Pattison, S. Manne, D. Carmean, K. Svore, M. Qureshi, and N. Delfosse, A scalable decoder micro-architecture for fault-tolerant quantum computing, ArXiv:2001.06598 (2020).

[106] M. Leifer and D. Poulin, Quantum graphical models and belief propagation, Ann. Phys. (NY) 323, 1899 (2008).

[107] D. Poulin and E. Bilgin, Belief propagation algorithm for computing correlation functions in finite-temperature quantum many-body systems on loopy graphs, Phys. Rev. A 77, 052318 (2008).

[108] D. Poulin and Y. Chung, On the iterative decoding of sparse quantum codes, Quantum Inf. Comput. 8, 0987 (2008).

[109] Y. Wang, B. C. Sanders, B. Bai, and X. Wang, Enhanced feedback iterative decoding of sparse quantum codes, IEEE Trans. Inf. Theory 58, 1231 (2012). 
[110] K.-Y. Kuo and C.-Y. Lai, Refined belief propagation decoding of sparse-graph quantum codes, IEEE J. Sel. Areas Inf. Theory 1, 487 (2020).

[111] N. Raveendran, M. Bahrami, and B. Vasic, in ICC 20192019 IEEE International Conference on Communications (ICC) (IEEE, Shanghai, China, 2019), p. 1.

[112] G. Duclos-Cianci and D. Poulin, Fast Decoders for Topological Quantum Codes, Phys. Rev. Lett. 104, 050504 (2010).

[113] A. Grospellier and A. Krishna, Numerical study of hypergraph product codes, ArXiv:1810.03681 (2018).

[114] A. Grospellier, L. Grouès, A. Krishna, and A. Leverrier, Combining hard and soft decoders for hypergraph product codes, ArXiv:2004.11199 (2020).

[115] J. Roffe, D. R. White, S. Burton, and E. T. Campbell, Decoding across the quantum LDPC code landscape, ArXiv:2005.07016 (2020).

[116] C. Liang, C. Cheng, Y. Lai, L. Chen, and H. H. Chen, Hardware-efficient belief propagation, IEEE Trans. Circuits Syst. Video Technol. 21, 525 (2011).

[117] J. Chen and M. P. Fossorier, Near optimum universal belief propagation based decoding of low-density parity check codes, IEEE Trans. Commun. 50, 406 (2002).

[118] N. Delfosse, V. Londe, and M. Beverland, Toward a union-find decoder for quantum LDPC codes, ArXiv:2103. 08049 (2021).

[119] N. Delfosse and M. B. Hastings, Union-find decoders for homological product codes, ArXiv:2009.14226 (2020).

[120] A. A. Kovalev, S. Prabhakar, I. Dumer, and L. P. Pryadko, Numerical and analytical bounds on threshold error rates for hypergraph-product codes, Phys. Rev. A 97, 062320 (2018).

[121] H. Bombín, Single-Shot Fault-Tolerant Quantum Error Correction, Phys. Rev. X 5, 031043 (2015).

[122] A. Grospellier, L. Grouès, A. Krishna, and A. Leverrier, Combining hard and soft decoders for hypergraph product codes, Quantum 5, 432 (2021).

[123] A. O. Quintavalle, M. Vasmer, J. Roffe, and E. T. Campbell, Single-shot error correction of three-dimensional homological product codes, ArXiv:2009.11790 (2020).

[124] M. B. Hastings, On quantum weight reduction, ArXiv:2102.10030 (2021).

[125] A. J. Kollár, M. Fitzpatrick, and A. A. Houck, Hyperbolic lattices in circuit quantum electrodynamics, Nature 571, 45 (2019).

[126] T. Bilski, Embedding graphs in books: A survey, IEE Proc. E (Comput. Dig. Tech.) 139, 134 (1992).

[127] V. Dujmović, A. Sidiropoulos, and D. R. Wood, Layouts of expander graphs, Chicago J. Theor. Comput. Sci. 16, 1 (2016).

[128] Qubit Zoo, https://www.qubitzoo.com/, accessed: 202102-02.

[129] A. G. Fowler, M. Mariantoni, J. M. Martinis, and A. N. Cleland, Surface codes: Towards practical large-scale quantum computation, Phys. Rev. A 86, 032324 (2012).

[130] C. Monroe and J. Kim, Scaling the ion trap quantum processor, Science 339, 1164 (2013).
[131] R. Nigmatullin, C. J. Ballance, N. De Beaudrap, and S. C. Benjamin, Minimally complex ion traps as modules for quantum communication and computing, New J. Phys. 18, 103028 (2016).

[132] N. H. Nickerson, J. F. Fitzsimons, and S. C. Benjamin, Freely Scalable Quantum Technologies using Cells of 5To-50 Qubits with Very Lossy and Noisy Photonic Links, Phys. Rev. X 4, 041041 (2014).

[133] T. Rudolph, Why i am optimistic about the siliconphotonic route to quantum computing, APL Photonics 2, 030901 (2017).

[134] T. Pellizzari, S. A. Gardiner, J. I. Cirac, and P. Zoller, Decoherence, Continuous Observation, and Quantum Computing: A Cavity Qed Model, Phys. Rev. Lett. 75, 3788 (1995).

[135] A. C. J. Wade, M. Mattioli, and K. Mølmer, Single-atom single-photon coupling facilitated by atomic-ensemble dark-state mechanisms, Phys. Rev. A 94, 053830 (2016).

[136] J. Conrad, C. Chamberland, N. P. Breuckmann, and B. M. Terhal, The small stellated dodecahedron code and friends, Philos. Trans. R. Soc. A: Math. Phys. Eng. Sci. 376, 20170323 (2018).

[137] A. Y. Kitaev, A. Shen, M. N. Vyalyi, and M. N. Vyalyi, Classical and Quantum Computation (American Mathematical Soc., Providence, US, 2002), Vol. 47.

[138] D. Aharonov and T. Naveh, Quantum NP-A survey, ArXiv:quant-ph/0210077 (2002).

[139] S. Arora and S. Safra, Probabilistic checking of proofs: A new characterization of NP, J. ACM (JACM) 45, 70 (1998).

[140] I. Dinur, The PCP theorem by gap amplification, J. ACM (JACM) 54, 12 (2007).

[141] M. B. Hastings, Trivial low energy states for commuting Hamiltonians, and the quantum PCP conjecture, Quantum Inf. Comput. 13, 0393 (2013).

[142] M. H. Freedman and M. B. Hastings, Quantum systems on non- $k$-hyperfinite complexes: A generalization of classical statistical mechanics on expander graphs, ArXiv:1301.1363 (2013).

[143] A. Anshu and C. Nirkhe, Circuit lower bounds for low-energy states of quantum code Hamiltonians, ArXiv:2011.02044 (2020).

[144] L. Eldar and A. W. Harrow, in 2017 IEEE 58th Annual Symposium on Foundations of Computer Science (FOCS) (IEEE Computer Society Conference Publishing Services, Washington DC, US, 2017), p. 427, ISSN: 02725428.

[145] D. Aharonov, I. Arad, and T. Vidick, Guest column: The quantum PCP conjecture, ACM Sigact News 44, 47 (2013).

[146] M. Freedman and M. B. Hastings, Building manifolds from quantum codes, ArXiv:2012.02249 (2020).

[147] N. Agarwal, K. Chandrasekaran, A. Kolla, and V. Madan, On the expansion of group-based lifts, SIAM J. Discr. Math. 33, 1338 (2019), publisher: Society for Industrial and Applied Mathematics. 